\title{
Oscillation Methods
}

1 Title

2 Methodological Considerations for Studying Neural Oscillations

3 running title: analysis of neural oscillations

4

5 Authors

6 Thomas Donoghue ${ }^{1}$, Natalie Schaworonkow ${ }^{1}$, \& Bradley Voytek ${ }^{1,2,3,4}$

7

8 Affiliations

9 'Department of Cognitive Science, University of California, San Diego

$10{ }^{2}$ Neurosciences Graduate Program, University of California, San Diego

$11{ }^{3}$ Halıcıoğlu Data Science Institute, University of California, San Diego

$12{ }^{4}$ Kavli Institute for Brain and Mind, University of California, San Diego

Corresponding Author

15 Thomas Donoghue: tdonoghue.research@gmail.com

Contributions

18 All authors contributed to designing the study. T.D and N.S did the analyses and created the figures. All

19 authors contributed to writing and editing the paper.

20

21

ORCIDs

Donoghue

0000-0001-5911-0472

Schaworonkow

0000-0002-2103-1178

Voytek

$0000-0003-1640-2525$

Conflicts of Interest

The authors declare no competing interests.

\section{Acknowledgements}

We would like to thank Ryan Hammonds for his contributions to the methods and software tools used for this report. This work was supported by research funding from the National Institute of General Medical Sciences grant R01GM134363-02, National Science Foundation grant BCS-1736028, and a UC San Diego 35 


\section{Materials Descriptions \& Availability Statements}

\section{Project Repository}

This project is also made openly available through an online project repository in which the code and data are made available, with step-by-step guides through the analyses.

\section{Datasets}

This project uses simulated data. Code to recreate the exact simulations used is included in the project repository.

\section{Software}

Code used and written for this project was written in the Python programming language ( $>=v 3.7)$.

53 All the code used within this project is deposited in the project repository and is made openly 54 available and licensed for reuse.

This project uses the following Python packages for simulating and analyzing neural data:

63

64

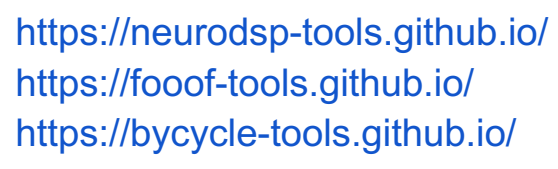

In addition, third party Python toolboxes including mne, numpy, scipy, matplotlib, and seaborn were used in this project. 


\section{methods for analyzing neural oscillations}

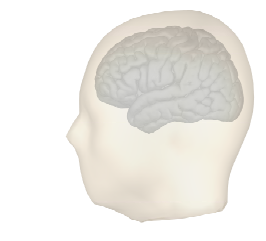

time domain

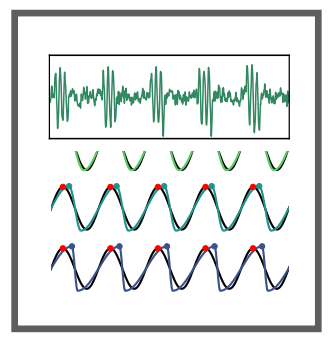

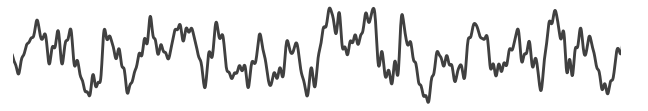

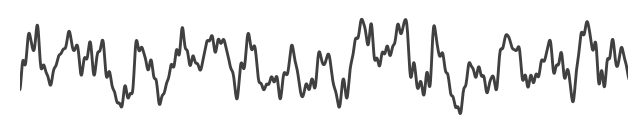

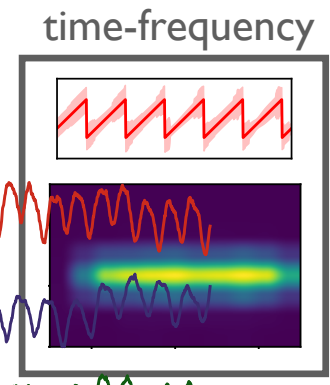

reviewing data properties \& method assumptions

Graphical Abstract. Neural oscillations are ubiquitous features of neural field data, with great potential for informing our understanding of neural function and how it relates to cognition. However, there is a great degree of variability in methods for investigating them, and findings that are reported. In this piece, we explore methodological considerations for analyzing neural oscillations, that may underlie some potential misinterpretations, and propose best practice guidelines for addressing them. 


\section{Oscillation Methods}

\section{Abstract}

Neural oscillations are ubiquitous across recording methodologies and species, broadly associated with cognitive tasks, and amenable to computational modeling that investigates neural circuit generating mechanisms and neural population dynamics. Because of this, neural oscillations offer an exciting potential opportunity for linking theory, physiology, and mechanisms of cognition. However, despite their prevalence, there are many concerns-new and old-about how our analysis assumptions are violated by known properties of field potential data. For investigations of neural oscillations to be properly interpreted, and ultimately developed into mechanistic theories, it is necessary to carefully consider the underlying assumptions of the methods we employ. Here, we discuss seven methodological considerations for analyzing neural oscillations. The considerations are to 1) verify the presence of oscillations, as they may be absent; 2) validate oscillation band definitions, to address variable peak frequencies; 3) account for concurrent non-oscillatory aperiodic activity, which might otherwise confound measures; measure and account for 4) temporal variability and 5) waveform shape of neural oscillations, which are often bursty and/or nonsinusoidal, potentially leading to spurious results; 6) separate spatially overlapping rhythms, which may interfere with each other; and 7) consider the required signal-to-noise ratio for obtaining reliable estimates. For each topic, we provide relevant examples, demonstrate potential errors of interpretation, and offer suggestions to address these issues. We primarily focus on univariate measures, such as power and phase estimates, though we discuss how these issues can propagate to multivariate measures. These considerations and recommendations offer a helpful guide for measuring and interpreting neural oscillations.

\section{Keywords}

neural field data, digital signal processing, electrophysiology, time series analysis, spectral analysis

\section{Abbreviations}

EEG: electroencephalography; MEG: magnetoencephalography; SNR: signal-to-noise ratio 


\section{Oscillation Methods}

\section{Introduction}

Recordings of electrical or magnetic fields in the brain, collectively referred to as neural field recordings, are commonly used for investigating links between physiology and behavior, cognition, and disease. A striking feature of such recordings is the prominent rhythmic activity, termed neural oscillations (Buzsáki \& Draguhn, 2004), that stands out in the otherwise seemingly chaotic activity of the brain. Neural oscillations have been a feature of interest since the early days of electrical brain recordings (Brazier, 1958), and are widely observed, being ubiquitously present across species (Buzsáki et al., 2013). Physiologically, field potential recordings largely reflect the aggregate postsynaptic and transmembrane currents of thousands to millions of neurons (Buzsáki et al., 2012), with neural oscillations thought to relate to population synchrony (Wang, 2010). As such, neural oscillations potentially offer insight into the coordination of neural activity at the population level. Theories of the functions of oscillations argue that they facilitate dynamic temporal and spatial organization of neural activity (Fries, 2005; VanRullen, 2016; Varela et al., 2001; Voytek \& Knight, 2015). Disruptions of oscillations have also been widely linked to neurological and psychiatric disease, and have been explored as potential biomarkers of disease status, drug efficacy, and other clinical indicators (Başar, 2013; Buzsáki \& Watson, 2012; Newson \& Thiagarajan, 2019).

Reflecting this broad interest, thousands of investigations conducted across many decades have reported associations between oscillations and just about every aspect of behavior and cognition that can be operationalized (Başar et al., 2001; Lopes da Silva, 2013; Mazaheri et al., 2018). As neural oscillations appear at many different temporal scales (Buzsáki et al., 2013), investigations often focus on predefined canonical frequency band ranges that are thought to capture distinct oscillations. For example, sleep researchers often study delta (1-4 Hz), memory researchers theta $(4-8 \mathrm{~Hz})$, visual researchers alpha $(8-12 \mathrm{~Hz})$, and cognitive and motor researchers beta $(13-30 \mathrm{~Hz})$ frequency bands. In doing so, research in neural oscillations spans across different recording modalities (Buzsáki et al., 2012)—including both non-invasive and invasive methods-and across different brain regions (Frauscher et al., 2018; Mahjoory et al., 2020).

While oscillations provide an exciting possibility to link cognition and disease to theory and physiology, there are often inconsistent reports regarding which oscillations are modulated by which conditions and how. In part, this likely reflects the variety of approaches taken, with limited consistency in terms of experimental design, analysis methods, parameter choices, and theoretical frameworks used across studies. Open challenges include developing more consistent 


\section{Oscillation Methods}

133 terminology and interpretations (Cohen \& Gulbinaite, 2014), and the need for explicitly

134 considering replicability in electrophysiological investigations (Cohen, 2017a). Accordingly, best

135 practice guidelines for research (Gross et al., 2013; Keil et al., 2014; Pernet et al., 2020; Pesaran

136 et al., 2018) and clinical investigations (Babiloni et al., 2020) have been proposed to improve 137 standards of reporting, and therefore reproducibility, for research using neural field recordings.

138 As an extension of these general guidelines, here we examine common interpretational 139 considerations in analyzing neural field recordings. Given the advances in both methods 140 development and our understanding of the empirical properties of the data under study, it is 141 critically important to ensure that common analysis methods are appropriately applied, as this is

142 a core requisite for accurate interpretation. There is a large toolkit of analysis methods for studying 143 neural oscillations, across both the spectral and temporal domains, borrowed and adapted from 144 the field of digital signal processing. These methods are described and compared in other work 145 focused on methodological properties of particular estimation techniques (Bruns, 2004; Gross, 2014; van Vugt et al., 2007; Wacker \& Witte, 2013).

Here, we focus more explicitly on properties of neural oscillations, and how these properties relate to commonly applied methods, rather than focus on the methods themselves. We address how common analysis approaches can give rise to results that are easy for researchers to misinterpret, due to the misalignment between methodological or experimental assumptions, and properties of the data. As such, these considerations are not restricted to individual estimators (such as using particular filters, or a particular estimate of power), as they reflect more general properties of signal processing methods and neural data. Importantly, these are not failures of the algorithms per se, which do, mathematically, exactly what they should; the potential pitfalls lay in how we interpret their outputs. If and when there is a misalignment between methodological assumptions and data properties, computed measures can lack validity which can lead to inconsistent results. This in turn impedes us from properly grounding oscillation research in physiology and theory.

To address these issues, we examine common interpretational considerations in studying neural oscillations, in order to identify and address possible methodological concerns that may lead to interpretation errors. We consider recurring themes based on our developing understanding of neural field data, and how this understanding relates to the application of 164 as a series of oscillatory signals, often assumed to be stationary. However, in empirical 165 neurophysiological data, oscillations show large variability in their presence and extent across 166 time, as well as across participants and cortical regions (Donoghue et al., 2020b; Frauscher et 


\section{Oscillation Methods}

167 al., 2018; Groppe et al., 2013). Even when oscillations are present, they are highly variable 168 (Jones, 2016; Neymotin, Barczak, et al., 2020), waxing and waning in short bursts and including 169 longer, more tonic rhythms, with rapidly changing amplitude, frequency, and phase dynamics that 170 are not easily captured by common analyses and predefined canonical frequency ranges. This 171 potentially meaningful variation of cycle features across time can be blurred by narrowband 172 filtering (de Cheveigné \& Nelken, 2019) and lead to misinterpretations of which features of the 173 oscillation have truly changed (Cole \& Voytek, 2019). All of these properties, and more, need to 174 be explicitly considered in order to accurately and reliably measure oscillatory neural activity.

175 We organize methodological considerations for analyzing neural oscillations into seven 176 areas, each with example demonstrations (see Box 1). The primary focus is on univariate 177 measures of oscillatory power, frequency, and phase, including potential pitfalls and 178 considerations for ensuring accurate measurement and interpretation of these aspects, as well 179 as discussions of how these issues can propagate to multivariate analyses, such as cross180 frequency coupling. These demonstrations make use of simulated data, which is created to match 181 known properties of neural field recordings whereby key features of the simulated neural field 182 activity were chosen and manipulated to reflect experimentally observed variations in empirical 183 data. We analyze the simulated data using common spectral and time-domain analysis methods 184 in order to evaluate their performance in relation to the interplay of data properties and method 185 assumptions. Each consideration is then contextualized within the broader literature, and specific 186 practical recommendations are made to help guide the analysis of neural oscillations. The 187 simulated data and analysis methods were created and used from the NeuroDSP module (Cole 188 et al., 2019), with all associated code for recreating and further exploring the illustrations openly 189 available in the project repository (https://github.com/voytekresearch/oscillationmethods). 
191 Box 1: Overview of methodological considerations for measuring neural oscillations

\begin{tabular}{|c|c|c|c|}
\hline Topic & Data Properties & Methodological Issues & Recommendation \\
\hline $\begin{array}{l}\text { \#1 } \\
\text { Oscillation } \\
\text { Presence }\end{array}$ & $\begin{array}{l}\text { neural oscillations are } \\
\text { variably present, and } \\
\text { may not be present in } \\
\text { the recording }\end{array}$ & $\begin{array}{l}\text { if there are no oscillations, } \\
\text { applied measures won't reflect } \\
\text { oscillatory activity, but will } \\
\text { return a value reflecting } \\
\text { aperiodic activity }\end{array}$ & $\begin{array}{l}\text { verify the presence of an } \\
\text { oscillation, such as with } \\
\text { spectral peak detection or with } \\
\text { burst detection in the time } \\
\text { domain }\end{array}$ \\
\hline $\begin{array}{l}\quad \# 2 \\
\text { Frequency } \\
\text { Variation }\end{array}$ & $\begin{array}{l}\text { neural oscillations } \\
\text { have variable peak } \\
\text { frequencies }\end{array}$ & $\begin{array}{l}\text { measures applied using } \\
\text { canonically defined frequency } \\
\text { bands may fail to accurately } \\
\text { capture oscillatory activity }\end{array}$ & $\begin{array}{c}\text { verify frequency ranges and } \\
\text { individualize as needed }\end{array}$ \\
\hline $\begin{array}{c}\text { \#3 } \\
\text { Aperiodic } \\
\text { Activity }\end{array}$ & $\begin{array}{l}\text { neural oscillations co- } \\
\text { exist with dynamic } \\
\text { aperiodic activity }\end{array}$ & $\begin{array}{l}\text { measured variation may arise } \\
\text { due to changes in aperiodic } \\
\text { activity, rather than changes in } \\
\text { oscillations }\end{array}$ & $\begin{array}{l}\text { measure and control for } \\
\text { changes in aperiodic neural } \\
\text { activity, evaluating whether it } \\
\text { explains measured changes }\end{array}$ \\
\hline $\begin{array}{c}\text { \#4 } \\
\text { Temporal } \\
\text { Variability }\end{array}$ & $\begin{array}{c}\text { neural oscillations are } \\
\text { variable across time, } \\
\text { exhibiting burst-like } \\
\text { properties }\end{array}$ & $\begin{array}{c}\text { burst properties may be } \\
\text { conflated when analyzing } \\
\text { spectral power, and trial } \\
\text { averages may suggest illusory } \\
\text { sustained activity }\end{array}$ & $\begin{array}{c}\text { examine single trial data for } \\
\text { temporal variation, and use } \\
\text { burst detection to evaluate } \\
\text { burst properties }\end{array}$ \\
\hline $\begin{array}{c}\text { \#5 } \\
\text { Waveform } \\
\text { Shape }\end{array}$ & $\begin{array}{l}\text { neural oscillations } \\
\text { have non-sinusoidal } \\
\text { waveform shape }\end{array}$ & $\begin{array}{l}\text { analysis methods often } \\
\text { assume sinusoidal structure, } \\
\text { and may return spurious } \\
\text { results in the case of non- } \\
\text { sinusoidal oscillations }\end{array}$ & $\begin{array}{c}\text { examine waveform shape } \\
\text { measures to evaluate if } \\
\text { waveform shape may underlie } \\
\text { the results }\end{array}$ \\
\hline $\begin{array}{l}\quad \text { \#6 } \\
\text { Overlapping } \\
\text { Oscillations }\end{array}$ & $\begin{array}{c}\text { multiple neural } \\
\text { oscillations co-exist } \\
\text { across the brain, and } \\
\text { may overlap across } \\
\text { space }\end{array}$ & $\begin{array}{l}\text { multiple distinct sources may } \\
\text { create destructive interference, } \\
\text { in which case measures won't } \\
\text { accurately reflect underlying } \\
\text { activity }\end{array}$ & $\begin{array}{l}\text { apply source separation } \\
\text { techniques in order to reduce } \\
\text { overlap of different types of } \\
\text { oscillations }\end{array}$ \\
\hline $\begin{array}{l}\text { \#7: } \\
\text { Signal-to- } \\
\text { Noise Ratio }\end{array}$ & $\begin{array}{l}\text { neural oscillations } \\
\text { have variable signal- } \\
\text { to-noise ratio }\end{array}$ & $\begin{array}{c}\text { without adequate signal to } \\
\text { noise ratio, measures may be } \\
\text { unreliable or inaccurate }\end{array}$ & $\begin{array}{c}\text { evaluate the required signal-to- } \\
\text { noise ratio, and potential ways } \\
\text { to optimize it for all applied } \\
\text { measures }\end{array}$ \\
\hline
\end{tabular}




\section{\#1 Neural oscillations are not always present}

\section{Why this matters}

Neural field recordings are characterized not only by oscillatory activity, but also aperiodic ' $1 / f$ ' or ' $1 / f-$-like' activity, in which signal power decreases exponentially as a function of frequency (Freeman et al., 2003; B. J. He, 2014). This is usually formalized as $1 / f^{x}$ where $x$ represents the decay of power across frequencies. In neural data, $x$ often ranges between 0 and 4 , where a signal with $X=0$ is white noise, with equal power across all frequencies, and higher values of $X$ indicate increasingly 'steeper' spectra. Aperiodic neural activity has been linked to the underlying activity of postsynaptic potentials and is a ubiquitous and sometimes dominant feature of neural field data (Gao et al., 2017; K. J. Miller et al., 2009).

The fact that aperiodic activity is omnipresent together with the large observable variability of neural oscillations (Donoghue et al., 2020b; Frauscher et al., 2018; Groppe et al., 2013) requires care in how band-limited power obtained by spectral analysis is measured and interpreted. Due to the presence of aperiodic activity, there is always non-zero power at all frequency bands. This means that any spectral measure-including computing a power spectrum, narrowband filtering, and average band-power measures-will always return a numerical value for power for a given frequency band, even if there is no oscillatory activity present. That is, just because there is power in a frequency band does not imply that there is an oscillation in that same frequency band (Bullock et al., 2003). It is a fallacy to presume that an analysis of a predefined narrowband frequency range necessarily reflects physiological oscillatory activity.

To introduce how transient and aperiodic signals are represented in the spectral domain, the Dirac delta can be used, whereby a single non-zero value in the time domain is represented by constant power across all frequencies in the frequency domain (Fig. 1A). This illustrates that power in a specific frequency band does not generally correspond to a present oscillation in the time domain. Similarly, 1/f-like aperiodic activity, which is common in neural data, shows power across all frequencies, with decreasing power for higher frequencies (Fig. 1B). Despite the lack of periodic activity in aperiodic time series, narrowband filtering, which imposes a sinusoidal basis, extracts components that appear to be oscillatory, when filtered into canonical band ranges (Fig. 1C). By comparison, rhythmic signals, such as a pure sinusoid, exhibit as a frequency specific peak in the power spectrum (Fig. 1D). Neural field recordings can be simulated as a summation of oscillatory and aperiodic components, resulting in a power spectrum that exhibits a spectral peak exceeding the aperiodic component, reflecting a high amount of band-specific power (Fig. 


\section{Oscillation Methods}

225 1E). In this case, the presence of the spectral peak is indicative of oscillatory power. In general,

226 since different signal components can contribute to spectral power across different frequency

227 ranges, power in a frequency band may not reflect oscillatory activity.

\section{Recommendations}

229 Investigations of oscillations should start with a detection step, verifying the presence of oscillations of interest. This verification step can be done in both the frequency and time domains. In the time domain, visualizing the data allows for examining if there are clear rhythmic segments in the data. In the frequency domain, oscillations manifest as peaks of power over and above the aperiodic signal (Buzsáki et al., 2013). As an initial check, visually inspecting power spectra can help to verify the presence of prominent oscillations. Including figures of power spectra in manuscripts is recommended, as it provides supporting evidence to the reader that there is oscillatory activity in the data under study.

Numerous quantitative methods also exist to detect oscillatory activity in neural field data, such as automated methods that detect narrowband spectral peaks (Pascual-Marqui et al., 1988). This can be systematically done by parameterizing the power spectrum, in which a mathematical model that quantifies periodic and aperiodic activity is applied to detect any putative oscillatory peaks above the measured aperiodic component (Donoghue et al., 2020b) (see Fig. 1F).

242 Similarly, both the 'multiple oscillation detection algorithm' (MODAL) method (Watrous et al., 243 2018) and the 'extended better oscillation detection' (eBOSC) method (Kosciessa et al., 2020), 244 which is itself an extension of prior methods (Caplan et al., 2015; Whitten et al., 2011), use a fit 245 of the aperiodic activity to detect frequency specific activity.

246 It may also be useful to examine rhythmic properties of the data, to search for putative 247 oscillatory activity in situations in which a spectral peak may be difficult to observe (Pesaran et 248 al., 2018). For example, oscillations may be present in the form of rare or infrequent bursts, which 249 will not appear as clear spectral peaks when the spectrum is calculated across the whole time 250 interval. In such situations, examining shorter time ranges, and selecting time windows with higher 251 band power and/or around events of interest may be required to resolve peaks in the frequency 252 domain. Alternatively, time domain and burst detection methods, further described in sections 4 253 and 5, may be more applicable. Another potential approach for addressing this is lagged 254 coherence (Fransen et al., 2015), which explicitly quantifies the rhythmicity in time series, in 255 contrast to measuring solely spectral power, and can also be used to differentiate between 256 oscillatory signals and transients (see Fig. 1A). 
Because oscillations can vary in their presence within and between participants, and 258 across different frequency bands (Donoghue et al., 2020b; Frauscher et al., 2018) oscillation 259 detection should be performed for each frequency band of interest, participant and analyzed 260 region. If oscillations are not detected, this may preclude further analyses. Group-level analyses 261 may obscure variation in oscillatory presence in individual participants. For example, if not all 262 participants display a clear rhythm, effect size estimates of oscillatory changes at the group level 263 may be confounded by including the subset of participants without any clear oscillatory activity. 264 Alternatively, a comparison of oscillatory power between regions without doing oscillation 265 detection may conflate a change in oscillatory power with a difference in oscillatory presence. 266 Analyses that include filtering or band-specific measures without first examining if an oscillation 267 is present can provide ambiguous results that may reflect aperiodic activity, in which case it is a 268 misinterpretation to describe physiological oscillatory activity. Applying analyses to detect 269 oscillatory presence can assure that measures reflect oscillatory activity. 


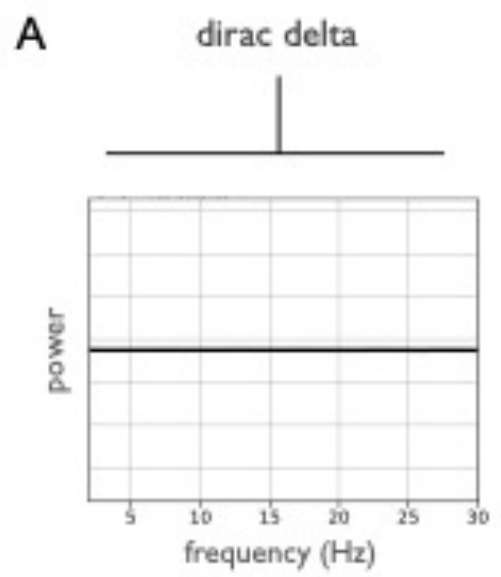

D

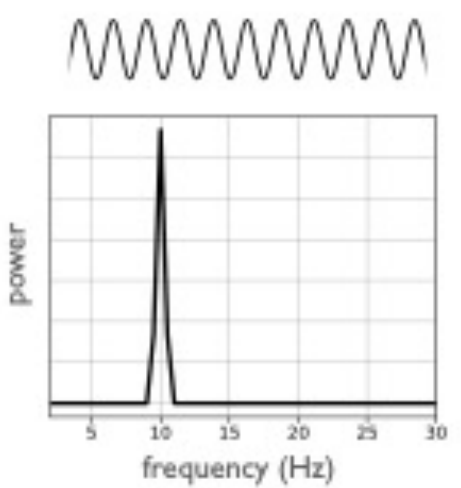

B aperiodic signal
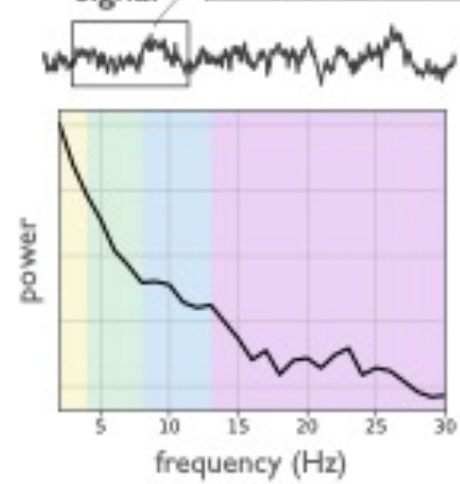

E

combined signal
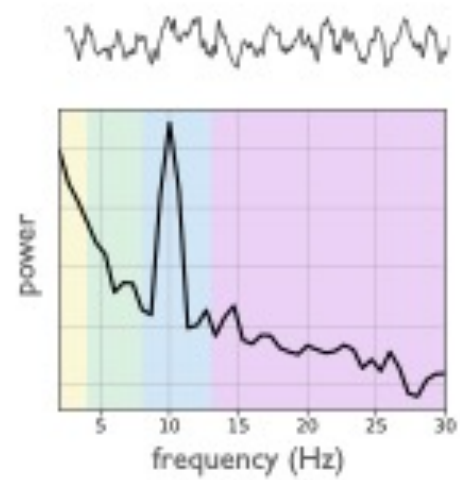

C filtered aperiodic activity

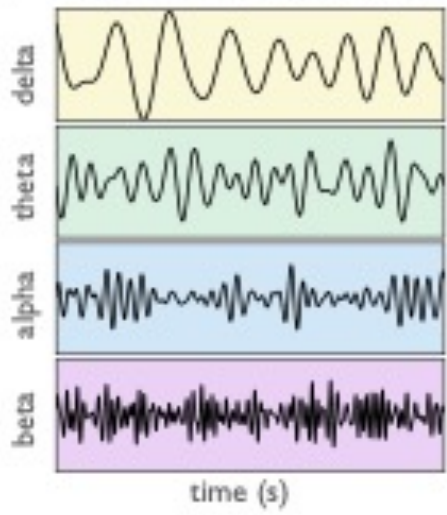

$\mathrm{F}$

spectral peak detection
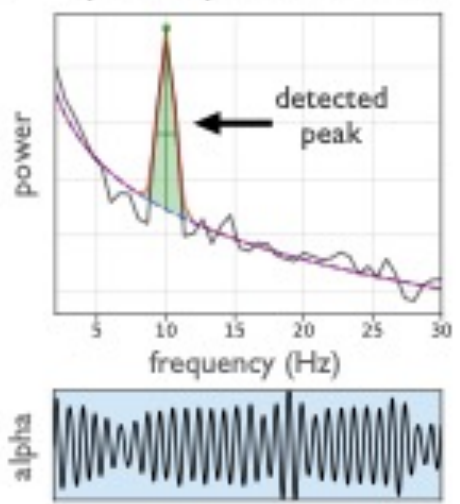

Figure 1: Without verified oscillatory activity, applied measures may reflect aperiodic activity. A) Non-oscillatory signals such as the dirac delta function exhibit power across all frequencies. B) Similarly, a non-oscillatory $1 / f$ signal also has power across all frequencies, including canonical narrowband regions: delta (yellow), theta (green), alpha (blue), and beta (purple). This power spectrum illustrates the fact that just because there is power at a frequency, that does not imply there is a dominant oscillation at that frequency. C) Narrowband filtered traces of the signal shown in B, that appear to be rhythmic. Note that this reflects band-power from the aperiodic activity, rather than any narrowband oscillation. D) Rhythmic signals, such as a pure sinusoid, exhibit as a dominant peak in the power spectrum. E) A combined signal that contains aperiodic activity and a narrowband alpha oscillation. In this case, the oscillation is visible as a peak in the power spectrum above the spectral contribution from the aperiodic $1 / f-$-like signal. F) Spectral peaks can be detected in order to identify putative oscillations in the data, as shown by the identified peak, in green. Spectral peak detection can be used to select frequency bands for further analysis, for example selecting the alpha range to be filtered for subsequent analysis (bottom). 


\section{Oscillation Methods}

\section{5 \#2 Neural oscillations vary in their peak frequencies}

286

287

288

289

290

291

292

293

294

295

296

297

298

299

300

301

302

303

304

305

306

307

308

309

310

311

312

313

314

315

316

\section{Why this matters}

Neural oscillations display significant variations in their peak frequencies, including variation across age (Lindsley, 1939), within and between participants (Haegens et al., 2014), and across cortical locations (Mahjoory et al., 2020). Alpha peak frequency, for example, is considered a stable trait marker (Grandy et al., 2013), and is also associated with some clinical disorders, displaying, for example, a slower frequency in attention-deficit hyperactivity disorder (ADHD) (Lansbergen et al., 2011). The frequency of neural oscillations can also vary within participants within a task (Benwell et al., 2019), including in task relevant ways (Wutz et al., 2018).

Due to frequency variation, even if the presence of oscillations is verified, the use of canonically defined frequency ranges may still fail to accurately reflect the data, as this may misestimate power of an oscillation if the spectral peak is not well captured in the canonical range. For example, in Figure 2, a canonically defined alpha range of 8-12 Hz captures the peak of a 10 $\mathrm{Hz}$ oscillation (Fig. 2A), but fails to accurately capture a $8 \mathrm{~Hz}$ peak (Fig. 2B). Despite the signals being simulated with the same amount of oscillatory power, estimated alpha power using a canonical frequency range differs between the signals (Fig. 2C), due to an underestimate of the power in the signal with an idiosyncratic peak frequency. This issue also impacts the result of band-pass filtering, as a canonical filter range underestimates the amount of alpha power present, as compared to an individualized band in which the filter range is made to reflect the oscillation in the data (Fig. 2D). Using individualized frequency band ranges to control for frequency differences accurately captures the alpha power in each signal (Fig. 2E). Overall, predefined frequency band definitions may fail to address variation in peak frequencies, and lead to misestimations.

Potential differences in peak frequency are important for analyses that compute an estimate within a specific frequency range, such as calculating band power, or narrowband filtering to a frequency range of interest. Applying a fixed frequency range may lead to information loss when the individual peak frequency lies near the border or outside of the defined range; it can also be non-specific if the range captures an adjacent oscillation or aperiodic activity. These issues apply both to analyses of individual frequency bands, as well as to composite measures such as ratios computed between the power of different frequency bands, since variation in the peak frequency or bandwidth of peaks can impact measured results (Donoghue et al., 2020a). For example, what had previously been reported as a difference in the theta / beta ratio of 


\section{Oscillation Methods}

317 participants with ADHD was found to be partially driven by a slowed alpha peak in the ADHD 318 group, changing the interpretation of the data (Lansbergen et al., 2011).

\section{Recommendations}

320 In order to address the variability of peak frequencies, any analyses that employ narrowband frequency ranges should assess how well the chosen ranges match the data. Visual inspection can help determine how well the defined frequency boundaries reflect actual peaks in the power spectra. This should be done for all analyzed frequency bands at the level of individual participants, because individual participants may have idiosyncratic peak frequencies that could influence group level results if they are misestimated. For within-subject analyses, changes in peak frequency over time or between tasks should also be considered in order to address whether a measured change in power could reflect a change in peak frequency, in which frequencies may 'drift' outside defined ranges of interest. Including power spectra in manuscripts also enables readers to observe that applied band ranges match the peaks observed in the data.

If canonically defined frequency ranges do appropriately match the data, then they can safely be used for subsequent analyses. However, if chosen band ranges of interest do not appropriately reflect the data, then individualized frequency bands may be applied (Klimesch, 1999). Methods for computing individualized frequency bands often do so by measuring spectral peaks (Haegens et al., 2014; Pascual-Marqui et al., 1988). Automated approaches have also been developed, that include spectral smoothing to improve performance (Corcoran et al., 2018). Such approaches don't always generalize to multiple peaks or bands, though some approaches use 'anchor frequencies' (Klimesch, 1999), defining, for example, theta as a range below the identified range of alpha. This approach has the limitation of not considering the oscillation detection step. Peak detection for multiple putative peaks, without predefining frequency ranges, can also be done with spectral parameterization (Donoghue et al., 2020b), after which peaks can be grouped into observed bands of interest.

Beyond spectral peak detection, methods for detecting oscillations can be used to detect

343 frequencies with peak rhythmicity, for example, by applying lagged coherence across frequencies

344 (Fransen et al., 2015). Some methods also allow for jointly learning multiple band definitions. For

345 example, the Oscillation ReConstruction Algorithm (ORCA) evaluates multiple band definitions in 346 terms of how well each definition is able to reconstruct the data (Watrous \& Buchanan, 2020), 347 and the gedBounds method identifies frequency boundaries by clustering similarities across 348 frequencies (Cohen, 2021). These methods, which examine all analyzed frequencies together, 349 may help to obtain more consistent groups of frequency ranges within and across participants. 
350 Collectively, some form of evaluation needs to be done to verify frequency bands, in order to 351 ensure that applied measures accurately capture the intended oscillatory activity.

352 


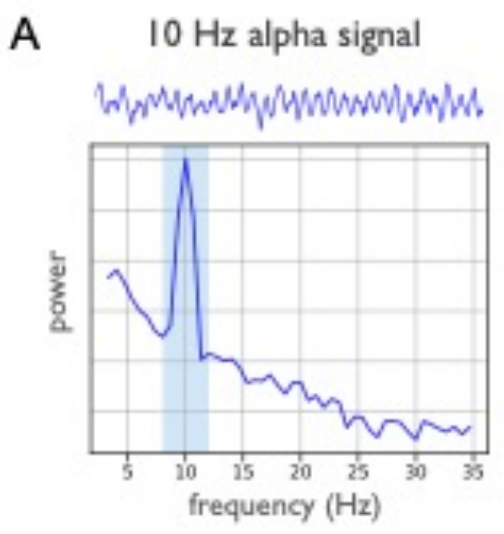

D filtered times series

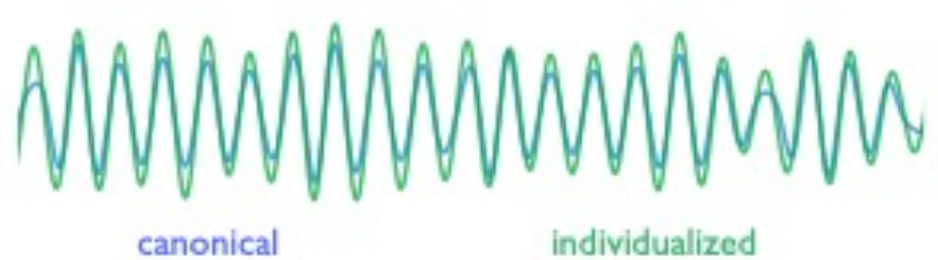

B

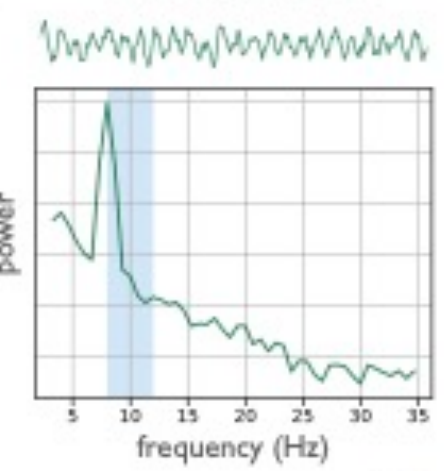

C canonical band powers

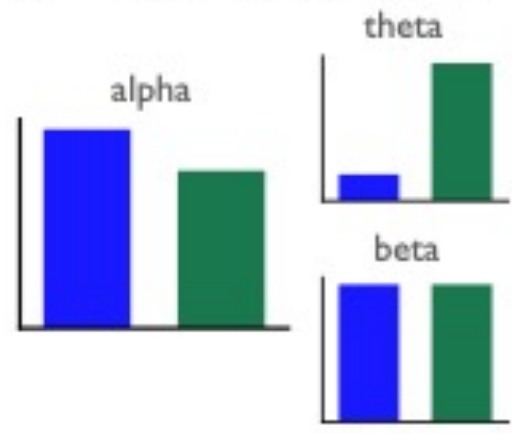

$\mathrm{E} \quad$ individualized band powers

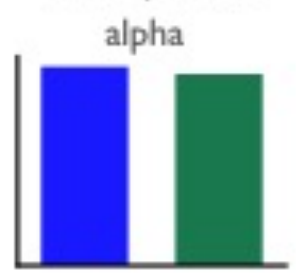

Figure 2: Canonical frequency band ranges may fail to capture narrowband peaks. A) A simulated signal, and corresponding power spectrum, with a prominent $10 \mathrm{~Hz}$ alpha oscillation. Shaded in blue is the canonical alpha range $(8-12 \mathrm{~Hz})$. B) Another signal with a prominent alpha oscillation, with a peak frequency of $8 \mathrm{~Hz}$. C) Using canonical band ranges, the amount of alpha power is found to be significantly different between the signals from $A \& B$. When examining adjacent frequency bands, (right), there is also a measured difference in theta power, due to the alpha peak drifting into the canonical theta range. These results suggest differences in oscillatory power between signals, however this is actually driven by a difference in alpha peak frequency. D) The time series from B, filtered into the alpha frequency range, using both the canonical range (blue) and an individualized range (green). The individualized range is tuned to the peak frequency of the time series (see inset power spectra). Note that the individualized filter captures more narrowband activity. E) Using individualized frequency bands, a difference in measured alpha power is no longer seen, consistent with the measured difference in $\mathrm{C}$ being due to a mismatch in peak frequency. 


\section{\#3 Neural oscillations coexist with aperiodic activity}

\section{Why this matters}

As previously introduced, neural field recordings contain aperiodic activity (B. J. He, 2014). This activity is not only ubiquitously present, but is also variable and dynamic within and between subjects (Freeman \& Zhai, 2009; Podvalny et al., 2015). Between subject variability of aperiodic activity can relate to age (W. He et al., 2019; Voytek et al., 2015), and clinical diagnoses (Robertson et al., 2019), whereas within subjects, aperiodic activity varies with state, such as sleep (Lendner et al., 2020), relates to behavioral tasks (Podvalny et al., 2015) and can be influenced by exogenous stimuli and cognitive demands (Waschke et al., 2021). This dynamic aperiodic activity has different putative generators, physiological interpretations, and task related dynamics (Gao et al., 2017, 2020; K. J. Miller et al., 2009, 2014), as compared to oscillations, making it an interesting feature of interest in itself. Altogether, aperiodic neural activity is dynamic in many contexts in which neural oscillations are usually the focus of inquiry.

This dynamic quality of aperiodic activity is an important consideration for detecting neural oscillations, as previously discussed (see \#1), as well as for interpreting measured changes in the data. With multiple dynamic components, analyses must adjudicate which aspects of the data are changing, and how, in order to allow for appropriate interpretations. Since aperiodic activity has power at all frequencies, changes or differences in aperiodic activity can induce patterns of differential activity across all frequencies. This can be seen by comparing white $(x=0)$ and pink $(x=1)$ noise $1 / f^{x}$ signals, which have different amounts of power in a canonically defined alphaband (Fig. 3A). Even with a validated spectral peak and frequency range, a difference in bandpower between two conditions within a given frequency range may not be specific to oscillatory changes, as it may reflect a global change in aperiodic activity. For example, in Fig. 3B, a measured difference in alpha-band power between two conditions reflects a change in the aperiodic exponent, not changes relating to a spectral peak in the alpha-band, since the periodic activity is the same in the two signals.

Considering aperiodic activity is particularly important for analyses that investigate bandpower across a series of frequency bands, since systematic patterns of measured changes across bands may not reflect any changes in oscillatory activity. For example, in Fig. 3C, the band-power of two conditions is compared across five different frequency bands. Despite this analysis suggesting a pattern of changes in band power across a series of canonically defined frequency bands (Fig. 3D), the changes are actually driven by a change in aperiodic activity. Patterns of 


\section{Oscillation Methods}

correlated changes across frequency bands can therefore sometimes be more parsimoniously explained by a change in aperiodic activity, rather than as multiple distinct oscillatory changes, as has been shown to be the case in development (W. He et al., 2019).

Changes in global power, due to aperiodic changes, can also impact relative or normalized measures of oscillatory activity. In the spectra in Fig. 3C, there is a visible spectral peak in the alpha-band. Even though there is no change in peak power, a relative power measure suggests a change in alpha power, due to a change in the power across all frequencies, that is driven by a change in aperiodic activity (Fig. 3E). This issue also impacts other compound measures, such as ratios of band-power, including the theta/beta-ratio, often investigated as a potential biomarker for ADHD (Lansbergen et al., 2011; Robertson et al., 2019), as it has been shown that band ratio measures often reflect a change of the aperiodic activity (Donoghue et al., 2020a), and that the putative relationship between $\mathrm{ADHD}$ and theta/beta-ratio appears to be driven by aperiodic activity (Robertson et al., 2019).

\section{Recommendations}

As both oscillatory and aperiodic components are dynamic, it is important for analyses to validate which elements of the data are specifically changing, in order to appropriately interpret results. This is relevant for any analysis investigating putative narrowband power, including investigations that examine multiple oscillation bands. Aperiodic activity should be explicitly measured to evaluate whether it explains the band-specific changes, including whether correlated patterns of changes across frequency bands may be more parsimoniously explained as a change in the broadband aperiodic activity. Approaches that assume oscillations exist upon a stationary 'background', such as relative power measures that divide by power across all frequencies, or band ratio measures, should be avoided, as they conflate changes in oscillatory and aperiodic components (Donoghue et al., 2020a). For example, a change in a relative power measure could arise from a change in band-specific power of interest, or be due to a change in aperiodic component that changes the measured power across all frequencies that is used as the denominator.

Explicitly measuring aperiodic activity requires methods that explicitly conceptualize both aperiodic and periodic activity, to avoid erroneously attributing aperiodic activity as oscillatory changes. Methods that define and measure oscillatory activity relative to aperiodic activity, including previously introduced methods such as spectral parameterization (Donoghue et al., 2020b) and eBOSC (Kosciessa et al., 2020), are designed to measure and control for aperiodic activity, and so address this issue. There are also dedicated methods for measuring aperiodic 
430 activity. For example, the irregular-resampling auto-spectral analysis (IRASA) method leverages 431 the scale-free nature of aperiodic activity by proposing a resampling procedure to isolate aperiodic 432 activity (Wen \& Liu, 2016). IRASA can be used to separate and measure aperiodic neural activity, 433 after which analyses can evaluate each component to examine whether measures of interest 434 specifically reflect the intended component. Overall, controlling for aperiodic activity requires 435 employing an oscillation detection step and evaluating oscillatory power relative to the aperiodic 436 component in order to assess whether measured changes are capturing oscillatory or aperiodic 437 activity.

438 
A

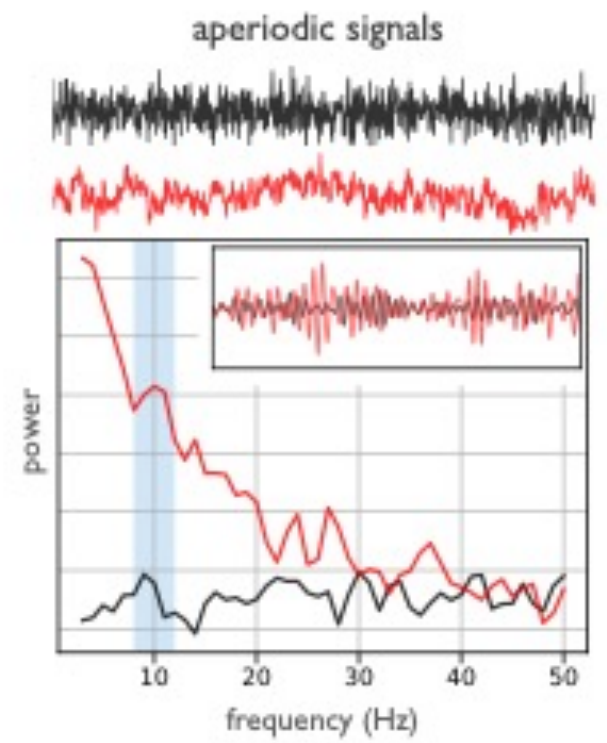

B

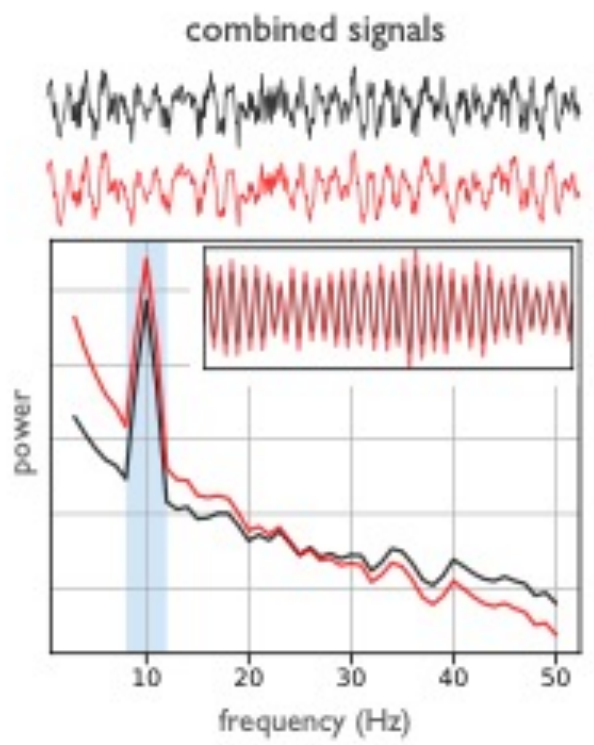

\section{C change in aperiodic activity}

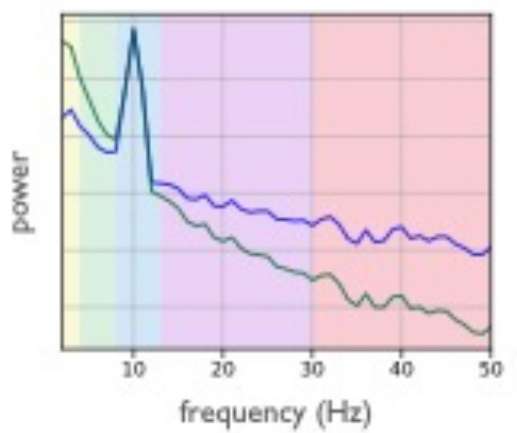

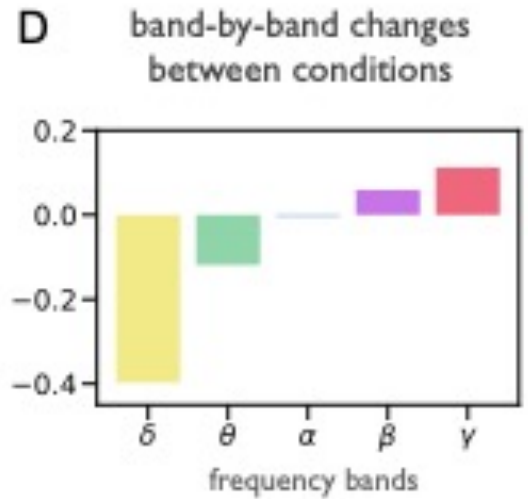

E relative power

absolute power power across all in alpha range frequencies
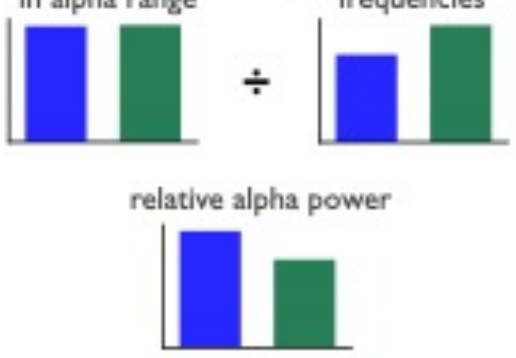

Figure 3: Variations in aperiodic activity influence band-power measures. A) Examples of aperiodic white (black) and pink (red) noise signals that display different patterns of power across frequencies, as seen in their power spectra. Shaded in blue is the canonical alpha range, with time-series filtered in the alpha-range shown in the inset. Note that the pink noise signal appears to have increased 'alpha' power. B) Simulated combined signals containing both aperiodic and oscillatory power (black), and a transformed version of the signal with the same periodic component with a change in the aperiodic component (red), after being rotated in the spectral domain. Note that in $A \& B$, what appears to be band-specific changes actually reflect differences in aperiodic activity. C) A comparison between power spectra for combined signals simulated with the same oscillatory component with different aperiodic activity. Shading reflects different frequency bands, including delta (yellow), theta (green), alpha (blue), beta (purple) and gamma (red). D) Absolute differences in power, calculated separately for each frequency band, for the spectra in C. Note that despite the difference in the data being simulated as a change in the aperiodic component, a band-by-band analysis suggests a pattern of changes across distinct frequency bands. E) Relative alpha power (bottom) is calculated as absolute band power (top left), divided by the power across all frequencies 455 (top right). Note that despite no difference in the amount of alpha power, there is measured change in relative power, due to systematically different aperiodic activity between the signals. 
Why this matters

Neural oscillations often display burst-like temporal dynamics (Lundqvist et al., 2016; Sherman et al., 2016) and are rarely, if ever, completely consistent and continuous. These temporal dynamics of neural oscillations are a potentially important feature; for example, the rate of burst events has been found to be predictive of behavior across tasks and species (Shin et al., 2017), including in investigations of working memory (Lundqvist et al., 2016) and motor activity (Wessel, 2020). Some generative models of oscillations predict non-continuous events in a way that is consistent with what is seen in empirical data (Sherman et al., 2016).

Despite this, many methods implicitly assume stationarity of the signal under study, when analyzing, for example, average band power across time or trials. In such cases, variability of oscillation presence or temporal dynamics can be misinterpreted as differences in power. For example, in simulations with stochastic onset and offset of oscillatory activity, signals can display different proportions of the data with oscillatory activity present, with the oscillatory power when present is equivalent (Fig. 4A). Measured power in such cases reports a different amount of band

472 specific power, typically interpreted as reflecting a change in the overall amplitude of the 473 oscillation, however, measured differences can be due to temporal variability (Fig. 4B). These

474 kinds of averaging effects are also important in scenarios such as time-frequency analyses that 475 average across trials, which may create an illusion of sustained activity in averaged data (Feingold 476 et al., 2015; Jones, 2016). This can happen if individual trials have burst-like temporal dynamics 477 that occur at different times across different trials, which can average together in a way as to 478 suggest a sustained response in average data, despite such continuity not occurring in any 479 individual trial (Fig. 4C). The temporal variability of neural oscillations motivates the importance 480 of considering single trial dynamics (Kosciessa et al., 2020; Rey et al., 2015; Stokes \& Spaak, 481 2016).

Oscillatory bursts can vary in multiple ways that can lead to similar measured changes in 483 band power, which may be misinterpreted as reflecting changes in tonic band power. This 484 includes changes in burst duration (Fig. 4D), burst occurrence (Fig. 4E), or burst amplitude (4F), 485 each of which can vary within or between analyzed time periods (Quinn et al., 2019; Zich et al., 486 2020). Understanding the different sources of variability has implications on how these signals 487 should be interpreted, as a change in the length, number, or size of bursts each likely reflect 488 different circuit mechanisms and putative relationships to neural function. However, this can not 


\section{Oscillation Methods}

489

490

491

492

493

494

495

496

497

498

499

500

501

502

503

504

505

506

507

508

509

510

511

512

513

514

515

516

517

518

519

be appropriately evaluated unless methods acknowledge oscillations as potentially transient, with potential variability in rate, timing, and duration as well as amplitude (van Ede et al., 2018).

\section{Recommendations}

Analyses of neural oscillations must therefore evaluate whether temporal variability, rather than overall power, may be driving measured changes. In order to address temporal variability, both the spectral and temporal domain have to be considered together (Zich et al., 2020). Timefrequency analyses, such as spectrograms, can be used to examine spectral properties across time in order to adjudicate between changes in the average power of oscillations and changes in their temporal dynamics. In doing so, it is important to analyze single-trials (Rey et al., 2015; Stokes \& Spaak, 2016), to avoid misinterpreting averaged power. If reporting spectrograms, single-trial examples should be included in order to evaluate whether apparent sustained activity is truly sustained, or arises as a result of averaging many short bursts.

Burst detection methods can also be applied to identify segments of the signal in which oscillations are present, which can then be characterized in terms of the durations of the bursts, the number of bursts, and/or the amplitude of the bursts. A common approach for burst detection is to use an amplitude threshold, detecting segments of power in which frequency specific power is greater than a chosen threshold level (Feingold et al., 2015). The previously described eBOSC algorithm (Kosciessa et al., 2020) can be considered to be a threshold based burst detection, in which the threshold is based on the aperiodic component, and can be used for burst detection.

Other algorithms for burst detection include matching pursuit, in which a dictionary of atoms, which can include oscillatory bursts, is fit to the data, providing potentially more accurate estimates of burst onset and duration (Chandran KS et al., 2018). Alternatively, methods such as hidden markov modelling can be used, which seek to characterize state transitions, and can be used to model transitions into and out of oscillatory states in a probabilistic way (Quinn et al., 2019; Vidaurre et al., 2016). Time-domain measures that identify oscillations by characterizing individual cycles, further described in \#5, can also be used to detect and analyze the number and duration of bursts, and their cycle-by-cycle properties (Cole \& Voytek, 2019; Schaworonkow \& Nikulin, 2019). After detection, analyses of burst-like neural activity typically involve subsequent analysis of the identified bursts, in order to evaluate whether they are changing in their duration, occurrence, and/or amplitude. 
A temporally variable time series

low burst probability time series

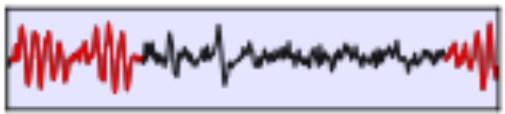

high burst probability time series

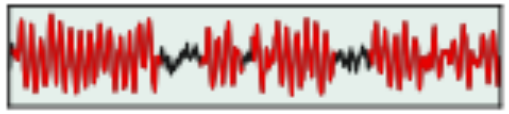

D burst duration

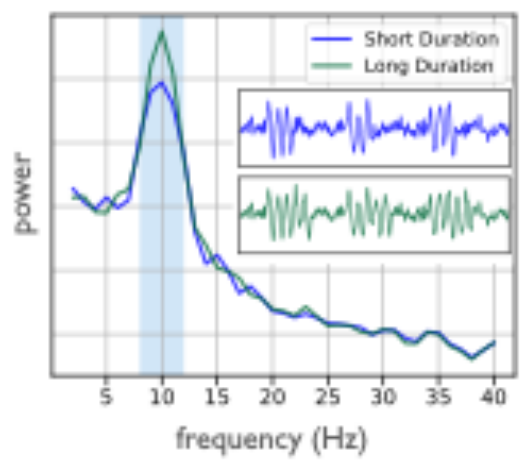

B spectral power of burst signals

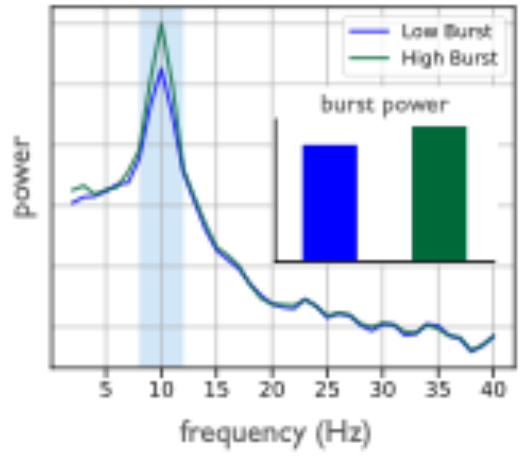

$\mathrm{E}$

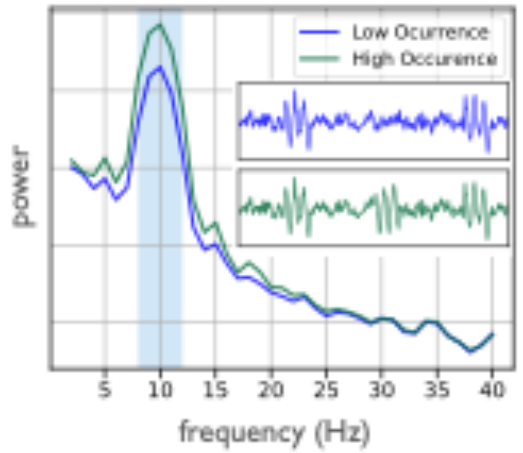

C average power of burst signals
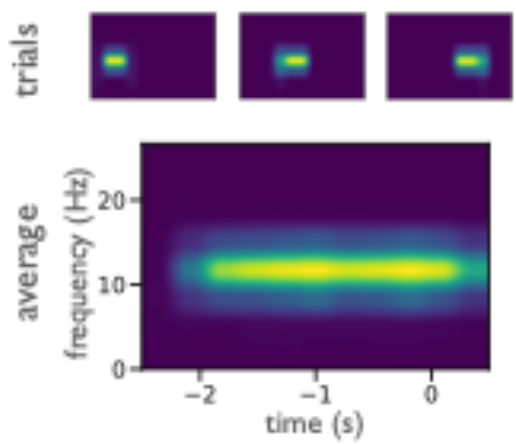

F burst amplitude

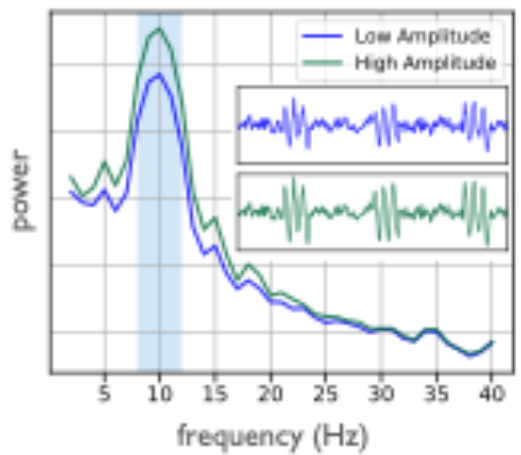

Figure 4: Temporal dynamics of neural oscillations influence spectral measures. A) Two simulated signals with lower (top; blue) and higher (bottom; green) levels of bursting activity in the alpha band, simulated with probabilistic burst onset and offset. Segments identified as bursts are shaded in red. Note that oscillation power, when present, is the same in both signals. B) Power spectra for the signals in A. Note the difference in size of the alpha peak, suggesting a difference in alpha power between the signals. However, when quantifying the power within the bursts (inset bar plot), the power is found to be approximately the same. The apparent difference in power is due to differences in temporal variability. C) Temporal variability can lead to spurious sustained power in averaged results. Spectrograms for individual trials (top) show short bursts of oscillatory power, which average to create what appears to be a sustained response (bottom). D-F) Measured differences in power can arise due to multiple features of bursting oscillations, including changes in the duration (D), occurrence (E), and/or amplitude (F) of the bursts. In these simulations, one feature differed between the two time series, while all others were held constant. Each feature creates a similar difference in the resultant alpha peaks, demonstrating that measured power 
Why this matters

The waveform shape of neural oscillations is often non-sinusoidal (Cole \& Voytek, 2017; Jones, 2016), as seen, for example, in the arc-shaped sensorimotor mu-rhythm, visual alpha, which can be triangular, and the sawtooth-shaped hippocampal theta-rhythm. These waveform properties of neural oscillations may reflect physiological properties, for example the

542 synchronization of neural activity (Schaworonkow \& Nikulin, 2019), spiking patterns of underlying 543 neurons (Cole \& Voytek, 2018), or behavioral correlates such as running speed (Ghosh et al., 544 2020). Waveform shape can therefore be an important feature of interest, with potential to impose 545 constraints on generative circuit models of oscillations (Sherman et al., 2016) as well as time 546 constants of involved synaptic currents.

547 The variable waveform shape of oscillations also creates substantial methodological and 548 interpretation hurdles, due to the assumed sinusoidal basis underlying most methods. For 549 instance, estimating instantaneous phase typically involves narrowband filtering the signal before 550 applying a Hilbert transform. Applying a narrowband filter on data with variations in waveform 551 shape can be problematic, as the phases of sinusoidal outputs of narrowband filtering will not 552 correspond to phases of a non-sinusoidal signal (Fig. 5A). This occurs because in the spectral 553 domain, nonsinusoidal shapes are represented by power across multiple frequencies, and if the 554 signal content in the harmonic frequencies is removed, the resulting filtered signal will have shifted 555 peaks and troughs compared to the original non-sinusoidal signal (Fig. 5A). This is an important 556 consideration for any analyses that examine cycle properties, such as the location of signal peaks 557 and troughs, as putatively corresponding to specific physiological states. For analyses that rely 558 on exact temporal characteristics (e.g. investigating the effects of pre-stimulus phase on behavioral measures), controlling for waveform shape may be beneficial.

In spectral analysis, non-sinusoidal waveforms are reflected in the power spectrum as harmonics occurring at multiples of the dominant frequency, as illustrated in Fig. 5B. This can 562 result in interpreting these separate peaks as independent physiological rhythms. In the case of 563 an arc-shaped mu-rhythm, for example, the waveform shape of the oscillation will create peaks 564 in both the alpha- and beta-frequency ranges. This may be interpreted as separate alpha- and 565 beta-rhythms with an assumed phase- and amplitude-coupled relationship, when in reality only 566 one non-sinusoidal rhythm is present. Differentiating between those situations is complicated by 567 the fact that several types of rhythms can be found across the cortex (see section \#6). Fig. 5C 


\section{Oscillation Methods}

568 shows how the degree of non-sinusoidality is reflected in the power of harmonic frequencies, with

569 higher power in the harmonic frequency range for increasing non-sinusoidality. This should be

570 considered when evaluating differences in spectral power between conditions, to control for 571 potential changes in waveform shape.

$572 \quad$ The spurious coupling that waveform shape can induce between frequencies (Kramer et 573 al., 2008) is especially important when considering measures such as phase-amplitude coupling 574 that are greatly influenced by waveform shape (Cole et al., 2017; Lozano-Soldevilla et al., 2016).

575 Waveform shape can result in systematic changes in the amplitude at harmonic frequencies, as 576 seen in Fig. 5D, which can depend on the phase of the base oscillation, as quantified in Fig. 5E. 577 This results in significant measures of cross-frequency phase-amplitude coupling. Numerically, 578 these values are not objectionable, as they reflect a relationship between frequencies in the 579 spectral domain. However, there is possible fallacy in the interpretation, if this relationship is taken 580 to reflect significant coupling between independent rhythms, when in fact no such interaction 581 between multiple rhythms need exist. Because of these methodological limitations, careful work 582 needs to be done to adjudicate between phase amplitude coupling measures that reflect 583 waveform shape versus those that truly reflect nested oscillations (Giehl et al., 2021; Vaz et al., 584 2017).

\section{Recommendations}

In order to evaluate and control for waveform shape, explicit measurement of waveform and cycle properties should be done. Time domain measures of individual cycles can be used to characterize waveform shape by, for example, calculating measures such as the rise/decay symmetry or peak sharpness (Cole \& Voytek, 2019; Schaworonkow \& Nikulin, 2019). Other methods aim at learning and grouping waveforms into observed categories, for example through attempting to learn recurring patterns in the data by sliding-window matching (Gips et al., 2017) 592 or by attempting to learn a dictionary of observed shapes in the data and finding occurrences of 593 particular waveforms in the data based on templates (Barthélemy et al., 2013; Brockmeier \& 594 Principe, 2016; Jas \& Dupré, 2017).

In the frequency domain, specific waveforms can create stereotypical patterns in power spectra and time-frequency representations, which can complicate the detection of oscillations

597 (see \#1). If spectral peaks are present at exact multiples of slower frequencies, quantifying 598 waveform shape may help to distinguish between an independent oscillation at that particular 599 frequency or harmonic spectral peaks induced by waveform shape. Since different waveform 600 shapes may exhibit similar time-frequency representations (Jones, 2016), time-domain analyses 


\section{Oscillation Methods}

601 may be required to evaluate if and how waveform shape is contributing to spectral 602 representations.

603 For cross-frequency coupling analysis, the frequency extent of local coupling within a 604 region (e.g., for phase amplitude coupling, the range of higher frequencies that are coupled to the 605 low frequency phase) can suggest whether it is likely to be genuine oscillatory coupling or a shape 606 effect (Cole et al., 2017; Vaz et al., 2017), with narrow ranges at exactly multiples of the base 607 frequencies indicative of possible coupling caused by waveform shape. Applying and comparing 608 multiple measures of cross-frequency coupling can dissociate harmonic and non-harmonic 609 phase-amplitude coupling (Giehl et al., 2021). More generally, frequency domain methods such 610 as bicoherence, a measure of non-linear interactions between frequencies, can also be used to 611 investigate waveform shape in the frequency domain (Bartz et al., 2019).

612 


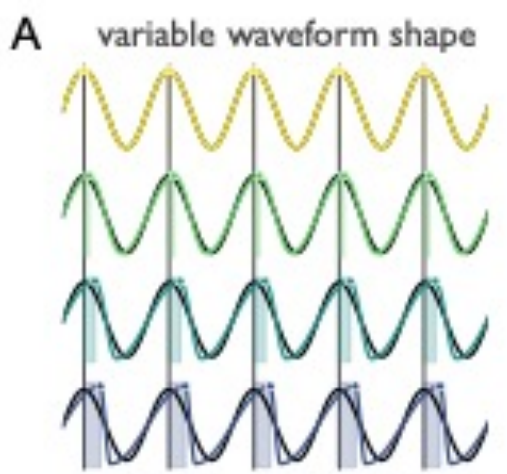

B harmonic spectral peaks

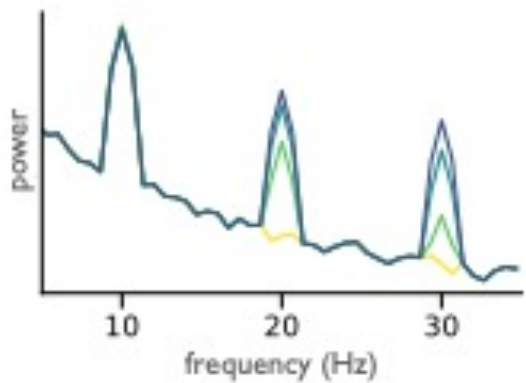

spurious harmonic power due to non-sinusoidal waveform shape

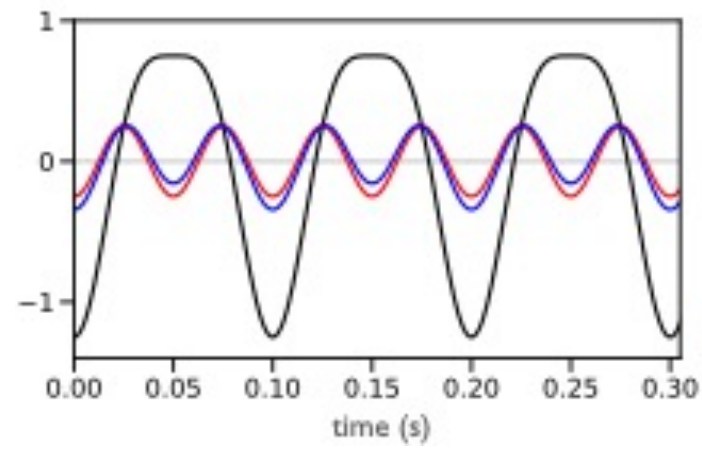

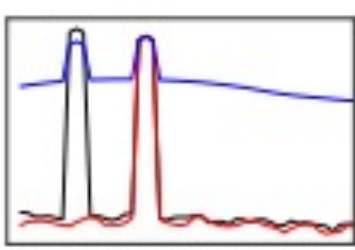

- non-sinuscidal waveform m sinusoid in beta band - bandpass filter (beta band)
C spurious beta power

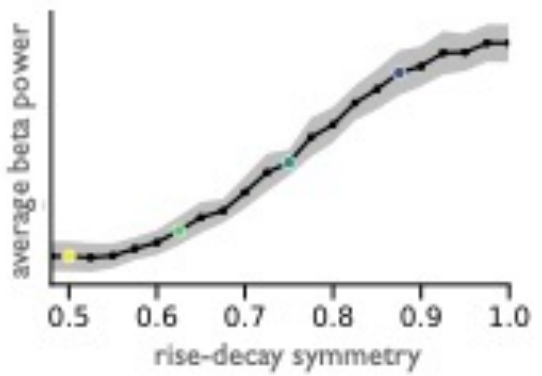

E phase-amplitude coupling

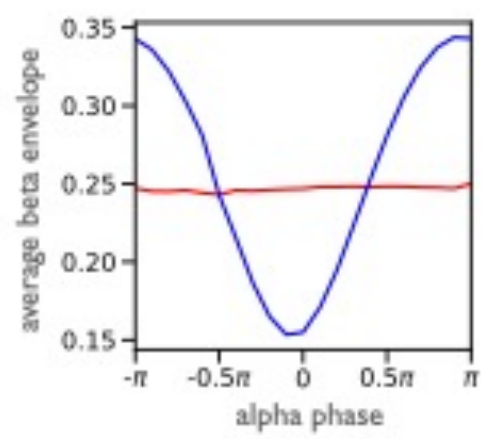

613

614

615

616

617

618

619

620

621

622

623

624

625

626

Figure 5: Waveform shape of neural oscillations influences power and coupling measures. A) Four different time domain signals with varying rise-decay asymmetry (colored traces) and their narrowband filtered versions (black traces). Narrowband filtering of asymmetric oscillations shifts the peak times of the signals as indicated by the shaded regions marking the distance between the peaks of original signal and the filtered version. B) In the corresponding power spectrum, there are emerging spectral peaks at harmonic frequencies (exactly two and three times the frequency) as a result of the asymmetry. $\mathbf{C}$ ) The scale of these harmonic peaks relates to the asymmetry, such that increasing waveform asymmetry can exhibit as increased power in the beta-frequency range. D) Non-sinusoidal rhythms can also create spurious phase amplitude coupling. A $10 \mathrm{~Hz}$ non-sinusoidal alpha signal is band-pass filtered around the beta peak frequency $(15-25 \mathrm{~Hz}$ ). The beta signal shows deviations in amplitude depending on alpha phase driven by the non-sinusoidal waveform shape (inset shows power spectra for each signal). E) Phase amplitude coupling is quantified by calculating beta envelope as a function of alpha phase. In contrast to a pure betasinusoid, the beta envelope from the non-sinusoidal signal shows a minimum for a specific alpha phase, indicating phase-amplitude coupling, which is driven by the waveform shape of the alpha rhythm. 


\section{\#6 Multiple oscillations coexist across the brain}

\section{Why this matters}

Non-invasive recordings of neural oscillations reflect aggregate activity across relatively large cortical areas. Through volume conduction, a term used to describe the propagation of electrical fields from their original source across tissues to recording sensors, recording electrodes can reflect activity from multiple local sources, as well contributions from more distant sources that overlap both spatially as well as temporally (Buzsáki et al., 2012; Nunez \& Srinivasan, 2006). For instance, in the context of MEG/EEG, there are several alpha-rhythm sources, with locations in somatosensory, occipital, parietal and temporal cortex (Hindriks et al., 2017), which can be co-active at the same time. In many studies, recordings are analyzed in sensor space, by directly analyzing activity from recording electrodes. In such cases, the aggregate signal may appear markedly different from the underlying sources of interest due to the spatial and temporal overlap of multiple distinct sources. Measures applied to these combined signals may therefore not accurately reflect the underlying sources, with distortions in measures of temporal dynamics or waveform shape (Schaworonkow \& Nikulin, 2019).

Examining how spectral and time domain measures can be affected by overlapping sources is shown in an example in which sensor space activity from a single electrode is composed of activity from two underlying sources in the parietal and visual cortices (Fig. 6A). In the spectral domain, this configuration can result in two peaks in the alpha-frequency range (Fig. $6 \mathrm{~B})$, when the two sources have slightly different peak frequencies. This has been observed in empirical data as 'double alpha' or 'split alpha' peaks (Chiang et al., 2008). Analyses in sensor space may lead to the interpretation that a specific circuit generates signals with two simultaneously present peak frequencies, which in turn will influence theories of generating mechanisms. Spatial summation of multiple underlying rhythms of similar peak frequencies can also mask temporal features of interest of the underlying rhythms, as seen in Fig. 6C, due to constructive and destructive interference effects (Schaworonkow \& Nikulin, 2019). Phase differences between sources of similar frequencies can attenuate the oscillation in sensor space, due to interference, even though oscillatory power has not changed in the underlying sources. This may lead to erroneous interpretations regarding changing oscillatory power of the sources, when it may be that only their relative temporal relationship has changed.

Inter-regional connectivity measures are also impacted by the simultaneous presence of multiple sources. Computing connectivity measures using sensor space signals can lead to 


\section{Oscillation Methods}

660 spurious findings, because volume conduction influences these measures (Haufe et al., 2013; J.

661 M. Palva et al., 2018; S. Palva \& Palva, 2012; Schoffelen \& Gross, 2009). Because individual

662 sources propagate to multiple sensors, regularities in amplitude and phase will be present across

663 multiple sensors. This can yield highly significant statistical relationships between electrodes,

664 reflecting signal content that is present due to a common source rather than genuine interregional 665 coupling, which may lead to erroneous interpretation of connectivity between oscillatory sources.

Recommendations

Due to overlapping sources, analyzing sensor level time series or power spectra can be misleading regarding which aspects of the oscillation are present and/or are changing. Whenever possible, sensor space analysis should be complemented by source-level analysis. Source separation methods can be applied to attempt to separate different narrowband periodic components in the signal, which can help to reveal features that are not visible in sensor space data, as well as helping to localize sources. There are many possible approaches for source separation. Because inferring the activity of many more sources than channels is not possible, constraints are needed to arrive at a specific decomposition. The choice of the appropriate method also depends on the specific goals of source separation, including, for example, localizing activity to specific regions and/or decomposing time series into components based on statistical properties.

Based on these goals, two main approaches with different optimization criteria can be used for estimating source activity from sensor space activity. The first main type of methods use anatomical information to constrain the inverse solution based on individual or template structural $\mathrm{MRI}$, in combination with methods such as beamformer or minimum norm estimation techniques 682 (Hauk et al., 2019). The second main type of methods are agnostic to anatomical information and 683 rely solely on the statistical structure of signals across channels. In this approach, channel activity 684 is assumed to be a linear mixture of multiple underlying sources, defined by a leadfield matrix, 685 which describes how individual sources map onto sensors (Parra et al., 2005). By assuming 686 specific statistical properties of the source time series as well as mixing properties, demixing can 687 be attempted. Methods in this realm include joint decorrelation (de Cheveigné \& Parra, 2014) or 688 independent component analysis (Hyvärinen \& Oja, 2000). In the context of investigating neural 689 oscillations, there are variants that specifically maximize SNR of narrowband oscillatory 690 components, while minimizing SNR in flanking bands or in comparison to broadband activity. For 691 enhancing oscillatory SNR, spatial-spectral decomposition (Nikulin et al., 2011) or generalized 692 eigendecomposition (Cohen, 2017b) can be used. The Common Spatial Patterns algorithm 
693 (Koles, 1991) and its variants (Lotte \& Cuntai Guan, 2011) can be used for maximizing differences 694 in narrowband activity between task conditions. For investigating relationships between 695 narrowband activity and a continuous variable, Source Power Correlation analysis (Dähne et al., 696 2014) may be of interest. Spatial filtering methods can also be used as a preprocessing step for 697 dimensionality reduction (Haufe, Dähne, et al., 2014), easing statistical comparisons and 698 computational needs.

699 Components that result from source separation need validation, since different methods 700 or parameter settings can yield highly different results, and solutions are not guaranteed to reflect 701 physiologically meaningful activity. As such, source separation can be non-trivial and has its own 702 set of methodological considerations as well as reporting guidelines (Cohen \& Gulbinaite, 2014; 703 Haufe, Meinecke, et al., 2014; Mahjoory et al., 2017). These guidelines can be used to evaluate 704 robustness of the solution, such as with goodness of fit and/or localization error metrics, and to 705 adequately convey reconstruction quality and method details to the reader. 706 
A

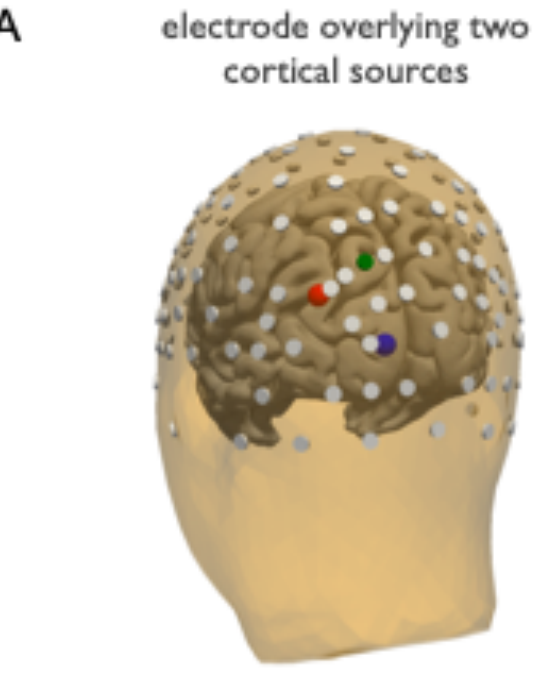

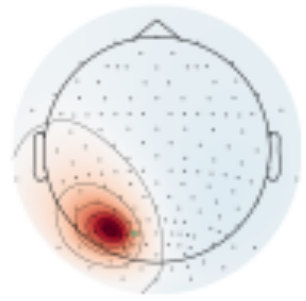

leadfield

source I

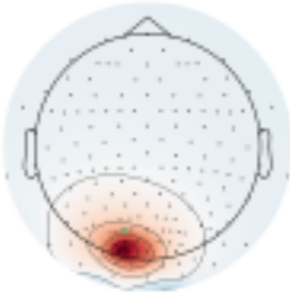

leadfield

source 2
B multiple spectral peaks due to source mixing

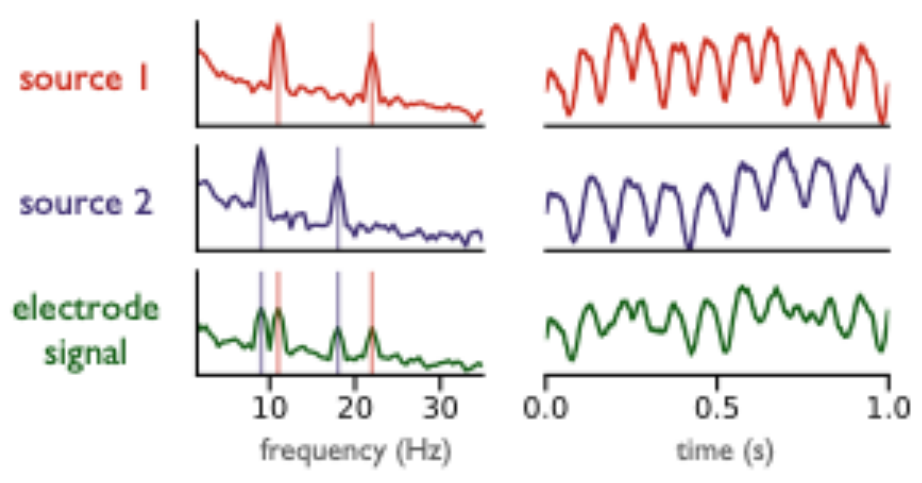

C time domain cancellation due to source mixing

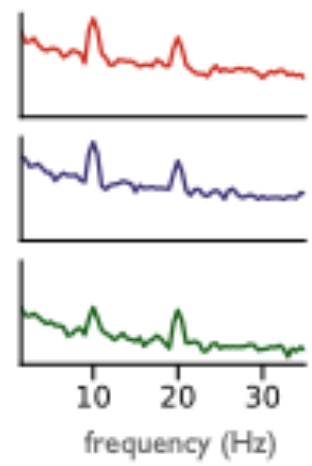

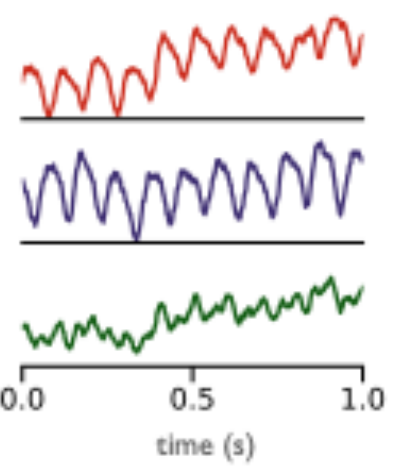

Figure 6: Multiple simultaneous rhythms can interfere and impact sensor level data. A) A realistic head model with two oscillatory sources (red and blue) placed in the posterior cortex which project on the highlighted electrode (green). Underneath are the topographies of the two sources that contribute to the recording electrode. The leadfield coefficients for the two sources have approximately equal values, indicating equal contribution to the activity recorded at the green electrode. B) In this simulation, the electrode signal (green; bottom) reflects multiple underlying sources, including two distinct rhythmic components, with slightly different peak frequencies. These sources can be seen as two spectral peaks in the power spectrum. C) A separate simulation of two oscillatory sources with the same peak frequency, with a phase difference. Due to a phase difference of pi, the two sources sum together destructively. In this scenario, interference of the sources cancel each other out at the electrode level, even though the oscillatory power of the individual sources is stable and consistent. 


\section{Oscillation Methods}

\section{8 \#7 Measures of neural oscillations require sufficient signal-to-noise ratio}

719

720

721

722

723

724

725

726

727

728

729

730

731

732

733

734

735

736

737

738

739

740

741

742

743

744

745

746

747

748

\section{Why this matters}

Neural oscillations are embedded in complex recordings containing multiple rhythmic signals, aperiodic activity, and transient events. Analyzing oscillatory signals of interest requires defining features of interest (signal), and extracting this signal from the rest of the data (noise).

As with all measures, methods for analyzing oscillations require an adequate signal to noise ratio (SNR). Indeed, ubiquitous processing steps such as filtering are used largely in order to increase the SNR (Widmann et al., 2015). Many of the considerations thus far (detecting oscillations, adjusting frequency ranges, controlling for aperiodic activity, burst detection, and source separation) can all be conceptualized as aiming to increase SNR by tuning analyses to specific properties of the data. Beyond these specific properties, applied measures can still be inaccurately estimated if SNR is low or variable.

The SNR of oscillatory activity relates to the ratio of oscillatory power to noise, typically the aperiodic background. Oscillatory power is a dynamic property, which can be seen by the variable height of oscillatory peaks over and above the aperiodic component (Fig. 7A). Many experimental paradigms will change oscillatory power, as presentation of stimuli may result in event-related attenuation of oscillations (Pfurtscheller \& Lopes da Silva, 1999). This change in oscillatory power changes SNR, which in turn may influence accuracy and stability of other oscillatory measures such as instantaneous phase and frequency. When SNR is high, estimations of phase and frequency can be reliably estimated (Fig. 7B). However, when SNR is low, estimation can be very noisy (Sameni \& Seraj, 2017) as can be seen in Fig. 7C, leading to artifactual large variations, often referred to as phase slips.

Changes in oscillatory power which change SNR and corrupt phase estimations can lead to inaccurate estimates of derived measures, such as the phase-locking value (Muthukumaraswamy \& Singh, 2011) or inter-trial coherence (van Diepen \& Mazaheri, 2018). Low SNR makes it difficult to reliably extract oscillations of interest (Fig. 7D), leading to variable phase estimates (Fig. 7E). When computing coupling measures on such estimates, differences in SNR, absent any true changes in phase alignment, can erode the detection of phase-locking between two signals (Fig. 7F). Unstable estimation of oscillatory measures can also propagate to multivariate analysis, such as cross-frequency coupling, whereby oscillatory power changes that influence SNR can lead to a change in measured cross-frequency coupling (Aru et al., 2015). 


\section{Oscillation Methods}

749 Time domain analyses, such as those designed for analyzing waveform shape, are also strongly

750 dependent on their being adequate SNR to meaningfully measure the properties of interest.

In cases of low SNR, unreliable estimates could, for example, lead to false-negatives due

752 to noisy estimations that are not able to adequately capture measures of interest. Conversely,

753 certain analyses may return false positive results, if the measured variability of the signal is mis-

754 interpreted as a feature of interest, and/or leads to an artifactual measured change between

755 conditions due to variable SNR. This may be an issue when comparing between groups who are

756 known to have differences in relative power of oscillations, and/or when comparing within

757 participants across conditions that may have different SNR.

\section{Recommendations}

Considering the SNR required for stable estimation of measures of oscillations starts by choosing appropriate experimental designs. When designing the protocol and tasks, experimenters should consider what is known about the reliability and effect size of effects of interest, and consider doing a power analysis to design well powered studies. This includes considering recording modalities, as different modalities have different sensitivities to different source locations (Piastra et al., 2020), as well as the different temporal, spatial, and frequency resolutions they offer. When recording the data, best practices should be employed to minimize non-neuronal noise, and use appropriate preprocessing in order to increase the quality of the data with the respect to desired analyses (Keil et al., 2014; Pernet et al., 2020).

Once recordings have been collected, or if considering existing datasets for potential reanalysis, signal-to-noise ratio has to be considered to validate if the dataset is appropriate for the desired analyses. This requires explicitly measuring SNR to verify that applied measures are robust in the SNR regime of the data. If the SNR is too low to provide accurate measurements,

772 the analyses may be non-viable, as any measurements will be uninterpretable. If the analysis can 773 be run, then SNR should still be continuously verified, to evaluate whether potential changes of

774 SNR across time or between conditions may explain measured changes in results (van Diepen \& 775 Mazaheri, 2018).

776 General approaches for optimizing SNR include good filter design (de Cheveigné \& 777 Nelken, 2019; Widmann et al., 2015), and using information about spectral estimators and signals 778 of interest to select the most appropriate methods to improve the accuracy and stability of 779 estimates (Chavez et al., 2006; Lepage et al., 2013). There are also specific methods for more 780 robust estimations of phase in low power situations, including Monte Carlo estimation (Sameni \& 781 Seraj, 2017) and applying a Kalman smoother (Mortezapouraghdam et al., 2018). Many of the 
782 previously described methods such as detecting oscillatory peaks, using individualized frequency 783 ranges, and using burst detection can all improve SNR. Source separation techniques, including

784 those that explicitly optimize SNR (Cheveigné \& Arzounian, 2015; Nikulin et al., 2011) can be 785 used to extract oscillatory components with higher SNR. 
A signals with variable SNR
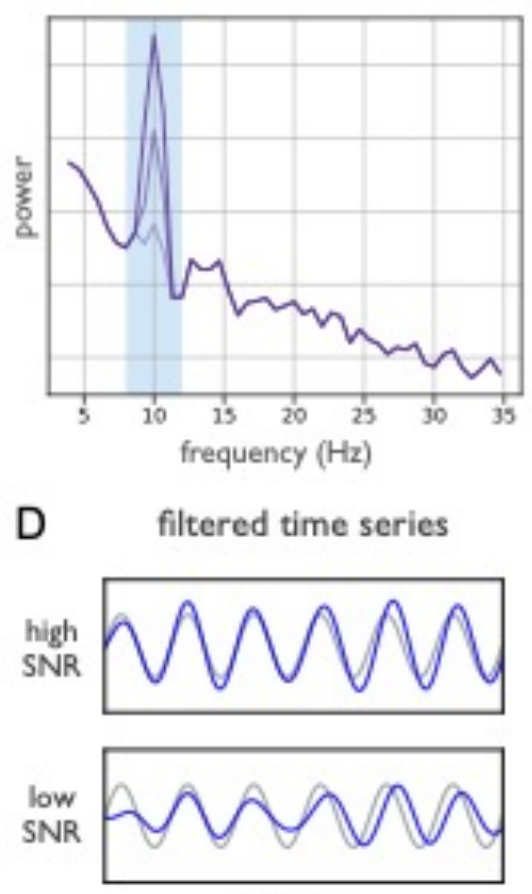

time (s)
B high SNR time series

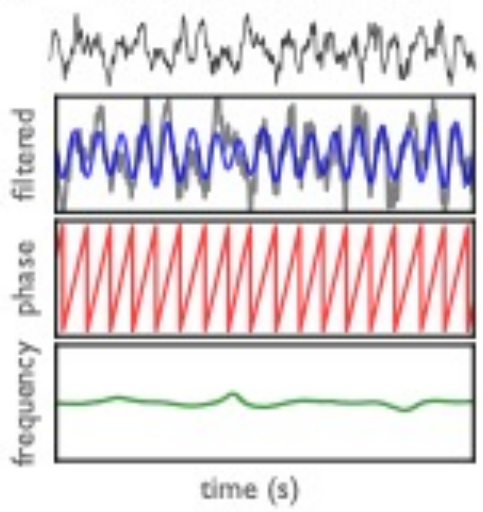

E

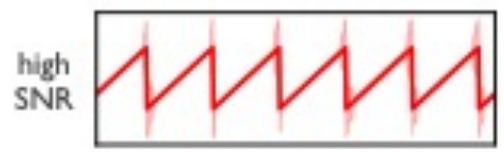

low

SNR

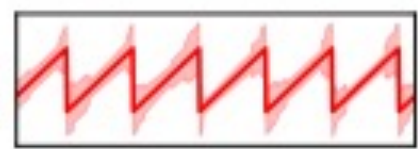

time (s)
C low SNR time series

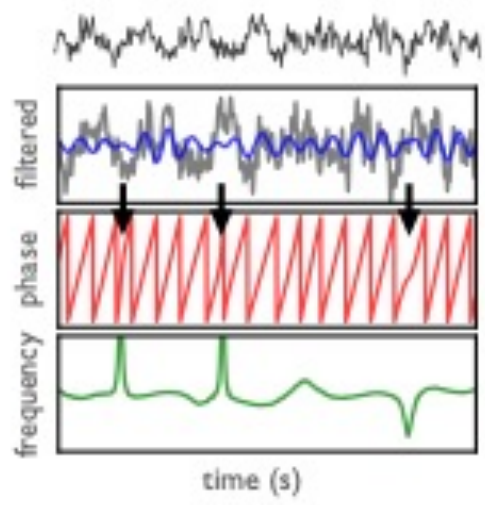

F phase locking value across oscillation SNR

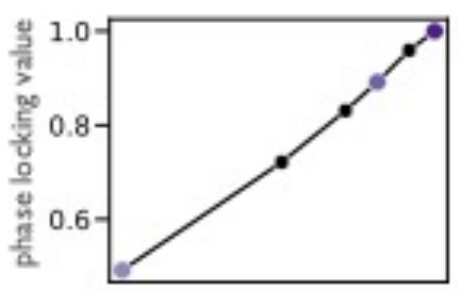

oscillation SNR

Figure 7: Low oscillatory signal-to-noise ratio impacts measures. A) Power spectra for simulated signals with variable SNR for an alpha oscillation, as seen in the different peak heights. B) One of the simulated signals, with a high SNR, with the alpha filtered signal (top; blue), from which the instantaneous phase (middle; red) and frequency (bottom; green) are computed. Note that the simulated signal has consistent phase and frequency. C) The same as B, for a signal with low SNR. Note that in this case, the estimates of phase and frequency are variable, due to misestimations because of the low SNR. This leads to phase slips, indicated by the arrows, in instantaneous phase, which also leads to erratic estimates of instantaneous frequency. D) Filtered versions of high and low SNR signals. In the simulated signals, the underlying signals (grey) are the same, other than a power difference, and have uniform phase. The filtered traces (blue) diverge from the underlying signal, especially in the low SNR signal. E) Phase estimates of the signals in $D$, in which the solid red is the true phase of the simulated oscillation, and the shading reflects the standard deviation of estimated phase across multiple iterations of phase estimation within each SNR regime. This shows that there is higher variance of phase estimates with lower SNR. These unstable phase estimates will impact subsequent measures, such as phase coupling. F) The phase locking value computed between a high powered oscillation, and simulated signals with decreasing power, as shown in A. Note that the simulated oscillations all have the same simulated phase time course, such that there is an expected phase locking value of 1 , and any estimates below this are misestimations due to low power. 


\section{Oscillation Methods}

804

805

806

807

808

809

810

811

812

813

814

815

816

817

818

819

820

821

822

823

824

825

826

827

828

829

830

831

832

833

834

835

836

\section{Discussion}

How, and to what extent, neural oscillations are mechanistically involved in cognition remains undetermined. This lack of clarity likely arises in part from imprecisions in our methodological approaches for analyzing oscillations that, in turn, give rise to inconsistent results. Here, we highlight specific methodological considerations for analyzing and interpreting neural oscillations, providing explicit recommendations regarding each topic. These considerations acknowledge the heterogeneity of neural oscillations and embrace this complexity as an opportunity to consider ideas and interpretations that may help us to further understand our data. Oscillations vary in their presence and frequency, co-exist with dynamic aperiodic activity, have idiosyncratic temporal and waveform shape properties, overlap with one another, and require sufficient SNR to appropriately analyze. These topics also demonstrate that there is an increasing set of features that can be defined for neural oscillations, with an increasing toolkit of estimation methods. Hopefully, these recommendations can serve as guidelines for potentially reducing misinterpretations and conflicting results, and can increase clarity in our understanding of neural oscillations.

These considerations relate broadly to studies investigating neural oscillations, including investigations of endogenous activity, and/or of rhythmic neural activity that may be induced by stimulus presentation (Doelling et al., 2019; Lakatos et al., 2008). The potential impact of the considerations may vary across different studies. In many cases, these considerations may not change the analyses or interpretations, but may still offer potential avenues for further analyses, and deeper understanding of the data. In some situations, these considerations may greatly impact results and interpretations, potentially reflecting fundamental confounds that do need to be addressed, or even reflect issues that cannot be addressed by current methods, such that it precludes particular analyses from being appropriately applied. Overall, with a range of possible impacts, the general recommendation is to check for all of these possible issues, to identify which topics may matter in each scenario, and proceed accordingly.

Though we present the considerations as seven distinct points, it is important to note that these considerations do not manifest in isolation from one another and can interact. For example, variable aperiodic activity (\#3) can interfere with spectral peak (\#1) and/or burst (\#4) detection, as it complicates approaches that use a threshold criterion to define bursts or spectral peaks. Oscillations may also be difficult to detect (\#1) and/or to individualize frequencies for (\#2) if they are temporally rare (\#4), and/or have low SNR (\#7). Further, waveform shape (\#5) may systematically vary in relation to underlying sources (\#6) (Schaworonkow \& Nikulin, 2019) and/or 


\section{Oscillation Methods}

837 detected peaks (\#1) may be volume conducted from remote sources (\#6), resulting, for example,

838 in 'double alpha' peaks due to the overlap of occipital and sensorimotor rhythms in the alpha-

839 band (Chiang et al., 2008). Multiple oscillatory features, such as power, waveform shape, burst

840 rate, etc., can covary. These potential multicollinearities need to be explicitly considered and

841 tested by robust analyses that control for multiple potentially confounding features by, for

842 example, addressing overlapping periodic and aperiodic activity (Donoghue et al., 2020b;

843 Kosciessa et al., 2020), controlling for waveform shape, which may result in spurious power-

844 and/or phase-coupling (Cole \& Voytek, 2019; Schaworonkow \& Nikulin, 2019), and examining

845 trial-by-trial dynamics that may be masked or conflated in average measures (Jones, 2016;

846 Stokes \& Spaak, 2016; Zich et al., 2020).

847 This investigation used a simulation approach that attempts to mimic the properties seen

848 in empirical data, including dynamic aperiodic activity, and oscillatory components that can vary 849 across multiple features (Cole et al., 2019). Because ground-truth properties of physiological data 850 are not known in a way that can be used to evaluate the accuracy of applied measures, simulated 851 data are an important tool for diagnosing available methods. In using simulated data, we must endeavor to reflect on our empirical data-simulating heterogeneous oscillatory features embedded within dynamic aperiodic activity-in order to be representative of empirical data and realistic use cases. As well as the tool used here, there are other approaches for simulating data, including for specific modalities such as EEG (Krol et al., 2018), or that emulate neural circuits (Neymotin, Daniels, et al., 2020), or whole brain recordings (Sanz Leon et al., 2013). Simulation analyses should be employed when developing new analysis approaches, as novel methods require validation and comparison to existing methods, such that best practice guidelines can be continuously developed and updated. All time-frequency methods include settings that should also be validated and explored. Sensitivity analyses, in which one repeats the analyses across mild perturbations of method settings to evaluate the robustness of the measured results, should be used to ensure that results are not overly dependent on specific parameter regimes.

Estimates of oscillatory features of interest are typically further analyzed and compared using statistical methods. Notably, many neuroscientific parameters exhibit skewed distributions

865 (Buzsáki \& Mizuseki, 2014), including oscillatory power (Kiebel et al., 2005). Therefore, 866 distributional properties of data should be carefully considered such that appropriate statistical 867 tests can be chosen (Maris, 2012; Maris \& Oostenveld, 2007). This is especially important when 868 considering that power-law distributed variables can result in spurious correlations when using 869 methods that assume normality (Schaworonkow et al., 2015). Statistical analyses, in particular in 870 the context of new methods and measures, should also evaluate consistency across participants 


\section{Oscillation Methods}

871 (Grice et al., 2020), reliability within participants, and effect size measures, which can be

872 computed using estimation statistics (Calin-Jageman \& Cumming, 2019). Considering effect sizes

873 can also aid in designing studies that are sufficiently powered (Button et al., 2013). Adopting the

874 best practices proposed here may also help to increase statistical power, insofar as they help to

875 better and more specifically characterize features of interest, improving SNR.

876 In our examples, we focused primarily on univariate measures, such as estimating 877 oscillatory power or phase. Issues that affect these estimates also propagate to derived 878 measures, such as correlations between amplitude or phase, as is done in functional connectivity 879 (Haufe et al., 2013) and cross-frequency coupling analyses (Aru et al., 2015). If phase estimates 880 are unreliable due to low oscillatory SNR (Sameni \& Seraj, 2017), or if amplitude estimates are 881 biased by changes in aperiodic activity (Donoghue et al., 2020b), or if burst properties vary 882 between analyzed signals (Jones, 2016), then derived measures may fail to reflect the intended oscillatory properties. Methodological limitations are likely to propagate and compound in multivariate or mass univariate analyses, and must therefore be considered for any analyses including, or built on top, of the univariate methods demonstrated here.

Though beyond the scope of this article, investigations of neural oscillations also require employing best-practices for designing, collecting, and preprocessing data in order to ensure sound research design, high quality data, and methodological validity. These considerations are covered in available textbooks (Cohen, 2014; Hari \& Puce, 2017), as well as individual reports that discuss topics such as including best practices for reporting and conducting MEG/EEG research (Gross et al., 2013; Keil et al., 2014; Pernet et al., 2020), pre-processing (de Cheveigné \& Arzounian, 2018), artifact rejection and data cleaning (Jas et al., 2017; Urigüen \& GarciaZapirain, 2015), and guides to using common software tools such as MNE (Gramfort, 2013; Jas et al., 2018) and FieldTrip (Oostenveld et al., 2011; Popov et al., 2018). Other work also features dedicated discussion for specific methods such as filtering (de Cheveigné \& Nelken, 2019; Widmann et al., 2015), phase estimations (Chavez et al., 2006; Lepage et al., 2013), functional connectivity (O’Neill et al., 2018), and cross-frequency coupling analyses (Aru et al., 2015).

Broader strategies are also required for addressing reproducibility in the field of neural oscillations, including pursuing replication studies, providing clear descriptions of methods and 900 results, and publishing null results (Cohen, 2017a). Open-science practices, including making 901 data and analysis code available, can help foster reproducibility and develop transparency 902 (Gleeson et al., 2017; Kathawalla et al., 2020; Voytek, 2016). Due to their computational nature, 903 investigations of neural oscillations also benefit from good code practice (Wilson et al., 2017). 904 Standardized procedures for organizing datasets also increase shareability, organization, and can 


\section{Oscillation Methods}

905 assist in standardized pipelines, making it easier to apply novel methods (Holdgraf et al., 2019;

906 Niso et al., 2018; Pernet et al., 2019). Adopting open science practices provides opportunities for

907 using open tools and datasets that can foster transparency and efficiently allow for revisiting the

908 evidence for how neural oscillations relate to cognition and disease.

909 Importantly, these considerations also reflect opportunities for developing new theory and 910 understanding of neural field data, which is still in many ways a mystery (Cohen, 2017c). Aperiodic 911 activity is itself a physiologically informative feature (Gao et al., 2017, 2020), reflecting processes

912 distinct from neural oscillations (Donoghue et al., 2020b; B. J. He, 2014). New methods provide

913 new opportunities, for example, the ability to jointly analyze multiple components of the data, such

914 as how oscillations and aperiodic activity jointly contribute to cognitive processing (Cross et al.,

915 2020). New features of interest offer the potential for better understanding underlying physiology

916 and putative computational roles of neural oscillations. For example, modelling that explicitly

917 considers waveform shape and/or burst properties has contributed to physiological models of

918 neocortical beta generation (Sherman et al., 2016), and models proposing mechanisms of beta

919 and gamma activity in working memory (E. K. Miller et al., 2018).

920 Our emerging understanding of the data under study and how to measure it provides new 921 vistas of opportunity for continuing to understand neural field data, and how it relates to cognition 922 and disease. These methods and topics reflect the current status of methodological

923 considerations for research related to neural oscillations. As our understanding of the many 924 complexities of neural data continues to evolve, future investigations of neural oscillations must 925 continue a consistent process of interrogating the assumptions of our methods and how they 926 relate to current knowledge of the data to validate measures of the data, and develop evolving 927 best practices. 
929 Conclusion

$930 \quad$ Productively investigating neural oscillations requires dedicated and carefully applied 931 methods that reflect our current understanding of the data. As methodological validity is a 932 prerequisite for appropriate interpretation, analysis methods must reflect that neural field data 933 consists of a complex combination of multiple oscillatory components, variable aperiodic activity, 934 and transient events, within which oscillations vary across multiple dimensions. Here, we have 935 proposed a checklist of methodological considerations for neural oscillations, with 936 recommendations to 1) validate that oscillations are present; 2) verify that used frequency ranges

937 are appropriate; 3 ) control for potential confounds due to aperiodic activity; consider the 4) 938 temporal variation and 5) waveform shape of neural oscillations; 6) apply source separation, as 939 needed, to separate multiple oscillatory processes; and 7) evaluate that the SNR is adequate for 940 the analyses at hand. These considerations, and new methods that have been developed to 941 address them, reflect our emerging understanding of neural field data and offer new possibilities 942 for investigating, and ultimately, understanding, neural oscillations. 


\section{Oscillation Methods}

\section{Materials and Methods}

945 A simulation-based approach was used to create the demonstrations in this manuscript. 946 Simulated time series were created with the NeuroDSP toolbox (Cole et al., 2019), version 2.2.0. 947 In most cases, the time series were created as a combination of oscillatory and aperiodic activity, 948 sampled at $1000 \mathrm{~Hz}$. Oscillatory activity was simulated as sine waves unless otherwise noted. 949 Each oscillation was simulated at a specific frequency, typically in the alpha band, unless 950 otherwise specified. Aperiodic activity was simulated by spectrally rotating white noise to the desired 1/f exponent (Timmer \& Konig, 1995). Aperiodic and oscillatory signal components were weighted according to a specified variance and combined together in an additive manner. Across all analyses, power spectra were estimated using Welch's method (Welch, 1967), using Hanning windowed 1 second segments with $12.5 \%$ overlap. Filtering was done with finite impulse response bandpass filters, with linear phase and filter lengths set to a default of 3 cycles of the highpass frequency, and enforced to be odd (Type I). Canonical band ranges were defined as delta (2-4 $\mathrm{Hz}$ ), theta $(4-8 \mathrm{~Hz})$, alpha $(8-13 \mathrm{~Hz})$, and beta $(13-30 \mathrm{~Hz})$, unless otherwise specified. Analysis methods were also used as available in the NeuroDSP toolbox, or with custom code included in the project repository (https://github.com/voytekresearch/oscillationmethods).

Several of the figure demonstrations used additional processing. For the peak detection in Figure 1, the spectral peak was detected and quantified using spectral parameterization, which models the power spectrum as a combination of aperiodic and oscillatory components, and can

963 be used to detects peaks of putative oscillatory power over and above the measured aperiodic 964 component (Donoghue et al., 2020b). For the individual frequency example in Figure 2, canonical 965 alpha was defined as $+/-2 \mathrm{~Hz}$ around $10 \mathrm{~Hz}$, and individualized alpha bands were defined as +/$9662 \mathrm{~Hz}$ around the individual peak frequency. For the demonstrations of varying aperiodic activity in

967 Figure 3, generated time series were spectrally rotated, in the same manner as done to simulate 968 the aperiodic activity (Timmer \& Konig, 1995). Relative power was computed as the sum of power 969 in a frequency band of interest, divided by the sum of power across all frequencies in the 970 frequency range of $2-50 \mathrm{~Hz}$.

971 For the temporal variation demonstrations in Figure 4, bursty oscillations were simulated 972 by specifying time segments that should include an oscillation, optionally controlling the duration, 973 occurrence, and amplitude of the bursts. Burst specific power was calculated by sub-selecting 974 segments of the data with an oscillation present. For the examinations of waveform shape in 975 Figure 5, oscillations were simulated as asymmetric sine waves, and the bycycle toolbox (version 976 1.0.0) was used to quantify waveform shape in the time domain (Cole \& Voytek, 2019). For this, 
977 signals were band-pass filtered around the frequency of interest (here: $10 \mathrm{~Hz}$ ) to extract the time 978 points of zero-crossings of the signal. The time points were used to segment the broadband data 979 into cycles, determining several cycle parameters. For this example, simulated time series were 980 created with varying rise-decay symmetry, which is the ratio of time in the rising and decaying 981 segments of the oscillation, which creates asymmetric oscillations.

982 For the spatial mixing demonstration in Figure 6, the New York Head (ICBM-NY) was used 983 (Huang et al., 2016) as a head model. Two sources are placed in the posterior cortex, and the 984 corresponding sensor signals are calculated using the leadfield. Oscillations were simulated as 985 asymmetric waves, created as the sum of two sines waves with a fixed phase lag. Topographies 986 were visualized using MNE-python (Gramfort, 2013). In Figure 7, instantaneous measures were 987 computed by applying the Hilbert transform to signals that had been bandpass filtered into the 988 alpha range $(8-12 \mathrm{~Hz})$, taking the angle as the phase estimate, and using the derivative of the 989 instantaneous phase as a measure of instantaneous frequency. Phase synchrony was measured 990 using the phase locking value (Lachaux et al., 1999). 
992

993

994

995

996

997

998

999

1000

1001

1002

1003

1004

1005

1006

1007

1008

1009

1010

1011

1012

1013

1014

1015

1016

1017

1018

1019

1020

1021

1022

1023

1024

1025

1026

1027

1028

1029

1030

1031

1032

1033

1034

1035

\section{References}

Aru, J., Aru, J., Priesemann, V., Wibral, M., Lana, L., Pipa, G., Singer, W., \& Vicente, R. (2015). Untangling cross-frequency coupling in neuroscience. Current Opinion in Neurobiology, 31, 51-61. https://doi.org/10.1016/j.conb.2014.08.002

Babiloni, C., Barry, R. J., Başar, E., Blinowska, K. J., Cichocki, A., Drinkenburg, W. H. I. M., Klimesch, W., Knight, R. T., Lopes da Silva, F., Nunez, P., Oostenveld, R., Jeong, J., Pascual-Marqui, R., Valdes-Sosa, P., \& Hallett, M. (2020). International Federation of Clinical Neurophysiology (IFCN) - EEG research workgroup: Recommendations on frequency and topographic analysis of resting state EEG rhythms. Part 1: Applications in clinical research studies. Clinical Neurophysiology, 131(1), 285-307. https://doi.org/10.1016/j.clinph.2019.06.234

Barthélemy, Q., Gouy-Pailler, C., Isaac, Y., Souloumiac, A., Larue, A., \& Mars, J. I. (2013). Multivariate temporal dictionary learning for EEG. Journal of Neuroscience Methods, 215(1), 19-28. https://doi.org/10.1016/j.jneumeth.2013.02.001

Bartz, S., Avarvand, F. S., Leicht, G., \& Nolte, G. (2019). Analyzing the waveshape of brain oscillations with bicoherence. Neurolmage, 188, 145-160. https://doi.org/10.1016/j.neuroimage.2018.11.045

Başar, E. (2013). Brain oscillations in neuropsychiatric disease. Dialogues in Clinical Neuroscience, 15(3), 291-300. https://doi.org/10.31887/DCNS.2013.15.3/ebasar

Başar, E., Başar-Eroğlu, C., Karakaş, S., \& Schürmann, M. (2001). Gamma, alpha, delta, and theta oscillations govern cognitive processes. International Journal of Psychophysiology, 39(2), 241-248. https://doi.org/10.1016/S0167-8760(00)00145-8

Benwell, C. S. Y., London, R. E., Tagliabue, C. F., Veniero, D., Gross, J., Keitel, C., \& Thut, G. (2019). Frequency and power of human alpha oscillations drift systematically with timeon-task. Neurolmage, 192, 101-114. https://doi.org/10.1016/j.neuroimage.2019.02.067

Brazier, M. A. B. (1958). The Development of Concepts Relating to the Electrical Activity of the Brain. The Journal of Nervous and Mental Disease, 126(4), 303-321.

Brockmeier, A. J., \& Principe, J. C. (2016). Learning Recurrent Waveforms Within EEGs. IEEE Transactions on Biomedical Engineering, 63(1), 43-54. https://doi.org/10.1109/TBME.2015.2499241

Bruns, A. (2004). Fourier-, Hilbert- and wavelet-based signal analysis: Are they really different approaches? Journal of Neuroscience Methods, 137(2), 321-332. https://doi.org/10.1016/j.jneumeth.2004.03.002

Bullock, T. H., Mcclune, M. C., \& Enright, J. T. (2003). Are the electroencephalograms mainly rhythmic? Assessment of periodicity in wide-band time series. Neuroscience, 121(1), 233-252. https://doi.org/10.1016/S0306-4522(03)00208-2

Button, K. S., loannidis, J. P. A., Mokrysz, C., Nosek, B. A., Flint, J., Robinson, E. S. J., \& Munafò, M. R. (2013). Power failure: Why small sample size undermines the reliability of neuroscience. Nature Reviews Neuroscience, 14(5), 365-376. https://doi.org/10.1038/nrn3475

Buzsáki, G., Anastassiou, C. A., \& Koch, C. (2012). The origin of extracellular fields and currents-EEG, ECoG, LFP and spikes. Nature Reviews Neuroscience, 13(6), 407-420. https://doi.org/10.1038/nrn3241 


\section{Oscillation Methods}

1036

1037

1038

1039

1040

1041

1042

1043

1044

1045

1046

1047

1048

1049

1050

1051

1052

1053

1054

1055

1056

1057

1058

1059

1060

1061

1062

1063

1064

1065

1066

1067

1068

1069

1070

1071

1072

1073

1074

1075

1076

1077

1078

1079

Buzsáki, G., \& Draguhn, A. (2004). Neural oscillations in cortical networks. Science, 304(5679), 1926-1929. https://doi.org/10.1126/science.1099745

Buzsáki, G., Logothetis, N., \& Singer, W. (2013). Scaling Brain Size, Keeping Timing: Evolutionary Preservation of Brain Rhythms. Neuron, 80(3), 751-764. https://doi.org/10.1016/j.neuron.2013.10.002

Buzsáki, G., \& Mizuseki, K. (2014). The log-dynamic brain: How skewed distributions affect network operations. Nature Reviews Neuroscience, 15(4), 264-278. https://doi.org/10.1038/nrn3687

Buzsáki, G., \& Watson, B. O. (2012). Brain Rhythms and Neural Syntax: Implications for Efficient Coding of Cognitive Content and Neuropsychiatric Disease. Dialogues in Clinical Neuroscience, 14(4), 345-367. https://doi.org/10.31887/DCNS.2012.14.4/gbuzsaki

Calin-Jageman, R. J., \& Cumming, G. (2019). Estimation for Better Inference in Neuroscience. Eneuro, 6(4), ENEURO.0205-19.2019. https://doi.org/10.1523/ENEURO.0205-19.2019

Caplan, J. B., Bottomley, M., Kang, P., \& Dixon, R. A. (2015). Distinguishing rhythmic from nonrhythmic brain activity during rest in healthy neurocognitive aging. Neurolmage, 112, 341-352. https://doi.org/10.1016/j.neuroimage.2015.03.001

Chandran KS, S., Seelamantula, C. S., \& Ray, S. (2018). Duration analysis using matching pursuit algorithm reveals longer bouts of gamma rhythm. Journal of Neurophysiology, 119(3), 808-821. https://doi.org/10.1152/jn.00154.2017

Chavez, M., Besserve, M., Adam, C., \& Martinerie, J. (2006). Towards a proper estimation of phase synchronization from time series. Journal of Neuroscience Methods, 154(1-2), 149-160. https://doi.org/10.1016/j.jneumeth.2005.12.009

Cheveigné, A. de, \& Arzounian, D. (2015). Scanning for oscillations. Journal of Neural Engineering, 12(6), 066020. https://doi.org/10.1088/1741-2560/12/6/066020

Chiang, A. K. I., Rennie, C. J., Robinson, P. A., Roberts, J. A., Rigozzi, M. K., Whitehouse, R. W., Hamilton, R. J., \& Gordon, E. (2008). Automated characterization of multiple alpha peaks in multi-site electroencephalograms. Journal of Neuroscience Methods, 168(2), 396-411. https://doi.org/10.1016/j.jneumeth.2007.11.001

Cohen, M. X. (2014). Analyzing neural time series data: Theory and practice. MIT Press.

Cohen, M. X. (2017a). Rigor and replication in time-frequency analyses of cognitive electrophysiology data. International Journal of Psychophysiology, 111, 80-87. https://doi.org/10.1016/j.ijpsycho.2016.02.001

Cohen, M. X. (2017b). Comparison of linear spatial filters for identifying oscillatory activity in multichannel data. Journal of Neuroscience Methods, 278, 1-12. https://doi.org/10.1016/j.jneumeth.2016.12.016

Cohen, M. X. (2017c). Where Does EEG Come From and What Does It Mean? Trends in Neurosciences, 40(4), 208-218. https://doi.org/10.1016/j.tins.2017.02.004

Cohen, M. X. (2021). A data-driven method to identify frequency boundaries in multichannel electrophysiology data. Journal of Neuroscience Methods, 347, 108949. https://doi.org/10.1016/j.jneumeth.2020.108949

Cohen, M. X., \& Gulbinaite, R. (2014). Five methodological challenges in cognitive electrophysiology. Neurolmage, 85, 702-710. https://doi.org/10.1016/j.neuroimage.2013.08.010 


\section{Oscillation Methods}

1080

1081

1082

1083

1084

1085

1086

1087

1088

1089

1090

1091

1092

1093

1094

1095

1096

1097

1098

1099

1100

1101

1102

1103

1104

1105

1106

1107

1108

1109

1110

1111

1112

1113

1114

1115

1116

1117

1118

1119

1120

1121

1122

1123
Cole, S. R., Donoghue, T., Gao, R., \& Voytek, B. (2019). NeuroDSP: A package for neural digital signal processing. Journal of Open Source Software, 4(36), 1272. https://doi.org/10.21105/joss.01272

Cole, S. R., van der Meij, R., Peterson, E. J., de Hemptinne, C., Starr, P. A., \& Voytek, B. (2017). Nonsinusoidal Beta Oscillations Reflect Cortical Pathophysiology in Parkinson's Disease. The Journal of Neuroscience, 37(18), 4830-4840. https://doi.org/10.1523/jneurosci.2208-16.2017

Cole, S. R., \& Voytek, B. (2017). Brain Oscillations and the Importance of Waveform Shape. Trends in Cognitive Sciences, 21(2), 137-149. https://doi.org/10.1016/j.tics.2016.12.008

Cole, S. R., \& Voytek, B. (2018). Hippocampal theta bursting and waveform shape reflect CA1 spiking patterns [Preprint]. bioRxiv. https://doi.org/10.1101/452987

Cole, S. R., \& Voytek, B. (2019). Cycle-by-cycle analysis of neural oscillations. Journal of Neurophysiology, 122(2), 849-861. https://doi.org/10.1152/jn.00273.2019

Corcoran, A. W., Alday, P. M., Schlesewsky, M., \& Bornkessel-Schlesewsky, I. (2018). Toward a reliable, automated method of individual alpha frequency (IAF) quantification. Psychophysiology, 55(7), e13064. https://doi.org/10.1111/psyp.13064

Cross, Z. R., Corcoran, A. W., Schlesewsky, M., Kohler, Mark. J., \& Bornkessel-Schlesewsky, I. (2020). Oscillatory and aperiodic neural activity jointly predict grammar learning [Preprint]. bioRxiv. https://doi.org/10.1101/2020.03.10.984971

Dähne, S., Meinecke, F. C., Haufe, S., Höhne, J., Tangermann, M., Müller, K.-R., \& Nikulin, V. V. (2014). SPoC: A novel framework for relating the amplitude of neuronal oscillations to behaviorally relevant parameters. Neurolmage, $86,111-122$. https://doi.org/10.1016/j.neuroimage.2013.07.079

de Cheveigné, A., \& Arzounian, D. (2018). Robust detrending, rereferencing, outlier detection, and inpainting for multichannel data. Neurolmage, 172, 903-912. https://doi.org/10.1016/j.neuroimage.2018.01.035

de Cheveigné, A., \& Nelken, I. (2019). Filters: When, Why, and How (Not) to Use Them. Neuron, 102(2), 280-293. https://doi.org/10.1016/j.neuron.2019.02.039

de Cheveigné, A., \& Parra, L. C. (2014). Joint decorrelation, a versatile tool for multichannel data analysis. Neurolmage, 98, 487-505. https://doi.org/10.1016/j.neuroimage.2014.05.068

Doelling, K. B., Assaneo, M. F., Bevilacqua, D., Pesaran, B., \& Poeppel, D. (2019). An oscillator model better predicts cortical entrainment to music. Proceedings of the National Academy of Sciences, 201816414. https://doi.org/10.1073/pnas.1816414116

Donoghue, T., Dominguez, J., \& Voytek, B. (2020a). Electrophysiological Frequency Band Ratio Measures Conflate Periodic and Aperiodic Neural Activity. Eneuro, ENEURO.019220.2020. https://doi.org/10.1523/ENEURO.0192-20.2020

Donoghue, T., Haller, M., Peterson, E. J., Varma, P., Sebastian, P., Gao, R., Noto, T., Lara, A. H., Wallis, J. D., Knight, R. T., Shestyuk, A., \& Voytek, B. (2020b). Parameterizing neural power spectra into periodic and aperiodic components. Nature Neuroscience, 23(12), 1655-1665. https://doi.org/10.1038/s41593-020-00744-x

Feingold, J., Gibson, D. J., DePasquale, B., \& Graybiel, A. M. (2015). Bursts of beta oscillation differentiate postperformance activity in the striatum and motor cortex of monkeys performing movement tasks. Proceedings of the National Academy of Sciences, 


\section{Oscillation Methods}

1124

1125

1126

1127

1128

1129

1130

1131

1132

1133

1134

1135

1136

1137

1138

1139

1140

1141

1142

1143

1144

1145

1146

1147

1148

1149

1150

1151

1152

1153

1154

1155

1156

1157

1158

1159

1160

1161

1162

1163

1164

1165

1166

1167
112(44), 13687-13692. https://doi.org/10.1073/pnas.1517629112

Fransen, A. M. M., van Ede, F., \& Maris, E. (2015). Identifying neuronal oscillations using rhythmicity. Neurolmage, 118, 256-267. https://doi.org/10.1016/j.neuroimage.2015.06.003

Frauscher, B., von Ellenrieder, N., Zelmann, R., Doležalová, I., Minotti, L., Olivier, A., Hall, J., Hoffmann, D., Nguyen, D. K., Kahane, P., Dubeau, F., \& Gotman, J. (2018). Atlas of the normal intracranial electroencephalogram: Neurophysiological awake activity in different cortical areas. Brain, 141(4), 1130-1144. https://doi.org/10.1093/brain/awy035

Freeman, W. J., Holmes, M. D., Burke, B. C., \& Vanhatalo, S. (2003). Spatial spectra of scalp EEG and EMG from awake humans. Clinical Neurophysiology, 114(6), 1053-1068. https://doi.org/10.1016/S1388-2457(03)00045-2

Freeman, W. J., \& Zhai, J. (2009). Simulated power spectral density (PSD) of background electrocorticogram (ECoG). Cognitive Neurodynamics, 3(1), 97-103. https://doi.org/10.1007/s11571-008-9064-y

Fries, P. (2005). A mechanism for cognitive dynamics: Neuronal communication through neuronal coherence. Trends in Cognitive Sciences, 9(10), 474-480.

https://doi.org/10.1016/j.tics.2005.08.011

Gao, R., Peterson, E. J., \& Voytek, B. (2017). Inferring synaptic excitation/inhibition balance from field potentials. Neurolmage, 158, 70-78. https://doi.org/10.1016/j.neuroimage.2017.06.078

Gao, R., van den Brink, R. L., Pfeffer, T., \& Voytek, B. (2020). Neuronal timescales are functionally dynamic and shaped by cortical microarchitecture. ELife, 9, e61277. https://doi.org/10.7554/eLife.61277

Ghosh, M., Shanahan, B. E., Furtak, S. C., Mashour, G. A., Burwell, R. D., \& Ahmed, O. J. (2020). Instantaneous Amplitude and Shape of Postrhinal Theta Oscillations Differentially Encode Running Speed. Behavioral Neuroscience, 134(6), 516-528.

Giehl, J., Noury, N., \& Siegel, M. (2021). Dissociating harmonic and non-harmonic phaseamplitude coupling in the human brain. Neurolmage, 227. https://doi.org/10.1016/j.neuroimage.2020.117648

Gips, B., Bahramisharif, A., Lowet, E., Roberts, M. J., de Weerd, P., Jensen, O., \& van der Eerden, J. (2017). Discovering recurring patterns in electrophysiological recordings. Journal of Neuroscience Methods, 275, 66-79. https://doi.org/10.1016/j.jneumeth.2016.11.001

Gleeson, P., Davison, A. P., Silver, R. A., \& Ascoli, G. A. (2017). A Commitment to Open Source in Neuroscience. Neuron, 96(5), 964-965. https://doi.org/10.1016/j.neuron.2017.10.013

Gramfort, A. (2013). MEG and EEG data analysis with MNE-Python. Frontiers in Neuroscience, 7, 267. https://doi.org/10.3389/fnins.2013.00267

Grandy, T. H., Werkle-Bergner, M., Chicherio, C., Schmiedek, F., Lövdén, M., \& Lindenberger, U. (2013). Peak individual alpha frequency qualifies as a stable neurophysiological trait marker in healthy younger and older adults: Alpha stability. Psychophysiology, 50(6), 570-582. https://doi.org/10.1111/psyp.12043

Grice, J. W., Medellin, E., Jones, I., Horvath, S., McDaniel, H., O’lansen, C., \& Baker, M. (2020). Persons as Effect Sizes. Advances in Methods and Practices in Psychological Science, 


\section{Oscillation Methods}

1168

1169

1170

1171

1172

1173

1174

1175

1176

1177

1178

1179

1180

1181

1182

1183

1184

1185

1186

1187

1188

1189

1190

1191

1192

1193

1194

1195

1196

1197

1198

1199

1200

1201

1202

1203

1204

1205

1206

1207

1208

1209

1210

1211
3(4), 443-455. https://doi.org/10.1177/2515245920922982

Groppe, D. M., Bickel, S., Keller, C. J., Jain, S. K., Hwang, S. T., Harden, C., \& Mehta, A. D. (2013). Dominant frequencies of resting human brain activity as measured by the electrocorticogram. Neurolmage, 79, 223-233. https://doi.org/10.1016/j.neuroimage.2013.04.044

Gross, J. (2014). Analytical methods and experimental approaches for electrophysiological studies of brain oscillations. Journal of Neuroscience Methods, 228, 57-66. https://doi.org/10.1016/j.jneumeth.2014.03.007

Gross, J., Baillet, S., Barnes, G. R., Henson, R. N., Hillebrand, A., Jensen, O., Jerbi, K., Litvak, V., Maess, B., Oostenveld, R., Parkkonen, L., Taylor, J. R., van Wassenhove, V., Wibral, M., \& Schoffelen, J.-M. (2013). Good practice for conducting and reporting MEG research. Neurolmage, 65, 349-363. https://doi.org/10.1016/j.neuroimage.2012.10.001

Haegens, S., Cousijn, H., Wallis, G., Harrison, P. J., \& Nobre, A. C. (2014). Inter- and intraindividual variability in alpha peak frequency. Neurolmage, 92, 46-55. https://doi.org/10.1016/j.neuroimage.2014.01.049

Hari, R., \& Puce, A. (2017). MEG-EEG Primer. Oxford University Press.

Haufe, S., Dähne, S., \& Nikulin, V. V. (2014). Dimensionality reduction for the analysis of brain oscillations. Neurolmage, 101, 583-597. https://doi.org/10.1016/j.neuroimage.2014.06.073

Haufe, S., Meinecke, F., Görgen, K., Dähne, S., Haynes, J.-D., Blankertz, B., \& Bießmann, F. (2014). On the interpretation of weight vectors of linear models in multivariate neuroimaging. Neurolmage, 87, 96-110. https://doi.org/10.1016/j.neuroimage.2013.10.067

Haufe, S., Nikulin, V. V., Müller, K.-R., \& Nolte, G. (2013). A critical assessment of connectivity measures for EEG data: A simulation study. Neurolmage, 64, 120-133. https://doi.org/10.1016/j.neuroimage.2012.09.036

Hauk, O., Stenroos, M., \& Treder, M. (2019). EEG/MEG Source Estimation and Spatial Filtering: The Linear Toolkit. In S. Supek \& C. J. Aine (Eds.), Magnetoencephalography (pp. 137). Springer International Publishing. https://doi.org/10.1007/978-3-319-62657-4_85-1

He, B. J. (2014). Scale-free brain activity: Past, present, and future. Trends in Cognitive Sciences, 18(9), 480-487. https://doi.org/10.1016/j.tics.2014.04.003

He, W., Donoghue, T., Sowman, P. F., Seymour, R. A., Brock, J., Crain, S., Voytek, B., \& Hillebrand, A. (2019). Co-Increasing Neuronal Noise and Beta Power in the Developing Brain (p. 49) [Preprint]. bioRxiv. https://doi.org/10.1101/839258

Hindriks, R., Micheli, C., Mantini, D., \& Deco, G. (2017). Human Resting-State Electrophysiological Networks In The Alpha Frequency Band: Evidence From Magnetoencephalographic Source Imaging [Preprint]. bioRxiv. https://doi.org/10.1101/142091

Holdgraf, C., Appelhoff, S., Bickel, S., Bouchard, K., D’Ambrosio, S., David, O., Devinsky, O., Dichter, B., Flinker, A., Foster, B. L., Gorgolewski, K. J., Groen, I., Groppe, D., Gunduz, A., Hamilton, L., Honey, C. J., Jas, M., Knight, R., Lachaux, J.-P., ... Hermes, D. (2019). IEEG-BIDS, extending the Brain Imaging Data Structure specification to human intracranial electrophysiology. Scientific Data, 6(1), 102. https://doi.org/10.1038/s41597019-0105-7 


\section{Oscillation Methods}

1212

1213

1214

1215

1216

1217

1218

1219

1220

1221

1222

1223

1224

1225

1226

1227

1228

1229

1230

1231

1232

1233

1234

1235

1236

1237

1238

1239

1240

1241

1242

1243

1244

1245

1246

1247

1248

1249

1250

1251

1252

1253

1254

1255
Huang, Y., Parra, L. C., \& Haufe, S. (2016). The New York Head-A precise standardized volume conductor model for EEG source localization and tES targeting. Neurolmage, 140, 150-162. https://doi.org/10.1016/j.neuroimage.2015.12.019

Hyvärinen, A., \& Oja, E. (2000). Independent component analysis: Algorithms and applications. Neural Networks, 13(4-5), 411-430. https://doi.org/10.1016/S0893-6080(00)00026-5

Jas, M., \& Dupré, T. (2017). Learning the Morphology of Brain Signals Using Alpha-Stable Convolutional Sparse Coding. Advances in Neural Information Processing Systems, 1099-1108.

Jas, M., Engemann, D. A., Bekhti, Y., Raimondo, F., \& Gramfort, A. (2017). Autoreject: Automated artifact rejection for MEG and EEG data. Neurolmage, 159, 417-429. https://doi.org/10.1016/j.neuroimage.2017.06.030

Jas, M., Larson, E., Engemann, D. A., Leppäkangas, J., Taulu, S., Hämäläinen, M., \& Gramfort, A. (2018). A Reproducible MEG/EEG Group Study With the MNE Software: Recommendations, Quality Assessments, and Good Practices. Frontiers in Neuroscience, 12, 530. https://doi.org/10.3389/fnins.2018.00530

Jones, S. R. (2016). When brain rhythms aren't 'rhythmic': Implication for their mechanisms and meaning. Current Opinion in Neurobiology, 40, 72-80. https://doi.org/10.1016/j.conb.2016.06.010

Kathawalla, U.-K., Silverstein, P., \& Syed, M. (2020). Easing Into Open Science: A Tutorial for Graduate Students [Preprint]. PsyArXiv. https://doi.org/10.31234/osf.io/vzjdp

Keil, A., Debener, S., Gratton, G., Junghöfer, M., Kappenman, E. S., Luck, S. J., Luu, P., Miller, G. A., \& Yee, C. M. (2014). Committee report: Publication guidelines and recommendations for studies using electroencephalography and magnetoencephalography: Guidelines for EEG and MEG. Psychophysiology, 51(1), 121. https://doi.org/10.1111/psyp. 12147

Kiebel, S. J., Tallon-Baudry, C., \& Friston, K. J. (2005). Parametric analysis of oscillatory activity as measured with EEG/MEG. Human Brain Mapping, 26(3), 170-177. https://doi.org/10.1002/hbm.20153

Klimesch, W. (1999). EEG alpha and theta oscillations reflect cognitive and memory performance: A review and analysis. Brain Research Reviews, 29(2), 169-195. https://doi.org/10.1016/S0165-0173(98)00056-3

Koles, Z. J. (1991). The quantitative extraction and topographic mapping of the abnormal components in the clinical EEG. Electroencephalography and Clinical Neurophysiology, 79(6), 440-447. https://doi.org/10.1016/0013-4694(91)90163-X

Kosciessa, J. Q., Grandy, T. H., Garrett, D. D., \& Werkle-Bergner, M. (2020). Single-trial characterization of neural rhythms: Potential and challenges. Neurolmage, 206, 116331. https://doi.org/10.1016/j.neuroimage.2019.116331

Kramer, M. A., Tort, A. B. L., \& Kopell, N. J. (2008). Sharp edge artifacts and spurious coupling in EEG frequency comodulation measures. Journal of Neuroscience Methods, 170(2), 352-357. https://doi.org/10.1016/j.jneumeth.2008.01.020

Krol, L. R., Pawlitzki, J., Lotte, F., Gramann, K., \& Zander, T. O. (2018). SEREEGA: Simulating event-related EEG activity. Journal of Neuroscience Methods, 309, 13-24. https://doi.org/10.1016/j.jneumeth.2018.08.001

Lachaux, J.-P., Rodriguez, E., Martinerie, J., Varela, F. J., \& others. (1999). Measuring phase 


\section{Oscillation Methods}

1256

1257

1258

1259

1260

1261

1262

1263

1264

1265

1266

1267

1268

1269

1270

1271

1272

1273

1274

1275

1276

1277

1278

1279

1280

1281

1282

1283

1284

1285

1286

1287

1288

1289

1290

1291

1292

1293

1294

1295

1296

1297

1298

1299

synchrony in brain signals. Human Brain Mapping, 8(4), 194-208.

https://doi.org/10.1002/(SICI)1097-0193(1999)8:4<194::AID-HBM4>3.0.CO;2-C

Lakatos, P., Karmos, G., Mehta, A. D., Ulbert, I., \& Schroeder, C. E. (2008). Entrainment of Neuronal Oscillations as a Mechanism of Attentional Selection. Science, 320(5872), 110-113. https://doi.org/10.1126/science.1154735

Lansbergen, M. M., Arns, M., van Dongen-Boomsma, M., Spronk, D., \& Buitelaar, J. K. (2011). The increase in theta/beta ratio on resting-state EEG in boys with attentiondeficit/hyperactivity disorder is mediated by slow alpha peak frequency. Progress in Neuro-Psychopharmacology and Biological Psychiatry, 35(1), 47-52. https://doi.org/10.1016/j.pnpbp.2010.08.004

Lendner, J. D., Helfrich, R. F., Mander, B. A., Romundstad, L., Lin, J. J., Walker, M. P., Larsson, P. G., \& Knight, R. T. (2020). An electrophysiological marker of arousal level in humans. ELife, 9, e55092. https://doi.org/10.7554/eLife.55092

Lepage, K. Q., Kramer, M. A., \& Eden, U. T. (2013). Some Sampling Properties of Common Phase Estimators. Neural Computation, 25(4), 901-921. https://doi.org/10.1162/NECO_a_00422

Lindsley, D. B. (1939). A Longitudinal Study of the Occipital Alpha Rhythm in Normal Children: Frequency and Amplitude Standards. The Pedagogical Seminary and Journal of Genetic Psychology, 55(1), 197-213. https://doi.org/10.1080/08856559.1939.10533190

Lopes da Silva, F. (2013). EEG and MEG: Relevance to Neuroscience. Neuron, 80(5), 11121128. https://doi.org/10.1016/j.neuron.2013.10.017

Lotte, F. \& Cuntai Guan. (2011). Regularizing Common Spatial Patterns to Improve BCl Designs: Unified Theory and New Algorithms. IEEE Transactions on Biomedical Engineering, 58(2), 355-362. https://doi.org/10.1109/TBME.2010.2082539

Lozano-Soldevilla, D., ter Huurne, N., \& Oostenveld, R. (2016). Neuronal Oscillations with Nonsinusoidal Morphology Produce Spurious Phase-to-Amplitude Coupling and Directionality. Frontiers in Computational Neuroscience, 10. https://doi.org/10.3389/fncom.2016.00087

Lundqvist, M., Rose, J., Herman, P., Brincat, S. L., Buschman, T. J., \& Miller, E. K. (2016). Gamma and Beta Bursts Underlie Working Memory. Neuron, 90(1), 152-164. https://doi.org/10.1016/j.neuron.2016.02.028

Mahjoory, K., Nikulin, V. V., Botrel, L., Linkenkaer-Hansen, K., Fato, M. M., \& Haufe, S. (2017). Consistency of EEG source localization and connectivity estimates. Neurolmage, 152, 590-601. https://doi.org/10.1016/j.neuroimage.2017.02.076

Mahjoory, K., Schoffelen, J.-M., Keitel, A., \& Gross, J. (2020). The frequency gradient of human resting-state brain oscillations follows cortical hierarchies. ELife, 9, e53715. https://doi.org/10.7554/eLife.53715

Maris, E. (2012). Statistical testing in electrophysiological studies: Statistical testing in electrophysiological studies. Psychophysiology, 49(4), 549-565. https://doi.org/10.1111/j.1469-8986.2011.01320.x

Maris, E., \& Oostenveld, R. (2007). Nonparametric statistical testing of EEG- and MEG-data. Journal of Neuroscience Methods, 164(1), 177-190. https://doi.org/10.1016/j.jneumeth.2007.03.024

Mazaheri, A., Slagter, H. A., Thut, G., \& Foxe, J. J. (2018). Orchestration of brain oscillations: 


\section{Oscillation Methods}

1300

1301

1302

1303

1304

1305

1306

1307

1308

1309

1310

1311

1312

1313

1314

1315

1316

1317

1318

1319

1320

1321

1322

1323

1324

1325

1326

1327

1328

1329

1330

1331

1332

1333

1334

1335

1336

1337

1338

1339

1340

1341

1342

1343

Principles and functions. European Journal of Neuroscience, 48(7), 2385-2388.

https://doi.org/10.1111/ejn.14189

Miller, E. K., Lundqvist, M., \& Bastos, A. M. (2018). Working Memory 2.0. Neuron, 100(2), 463475. https://doi.org/10.1016/j.neuron.2018.09.023

Miller, K. J., Honey, C. J., Hermes, D., Rao, R. P., den Nijs, M., \& Ojemann, J. G. (2014). Broadband changes in the cortical surface potential track activation of functionally diverse neuronal populations. Neurolmage, 85, 711-720.

https://doi.org/10.1016/j.neuroimage.2013.08.070

Miller, K. J., Sorensen, L. B., Ojemann, J. G., \& den Nijs, M. (2009). Power-Law Scaling in the Brain Surface Electric Potential. PLOS Computational Biology, 5(12), e1000609. https://doi.org/10.1371/journal.pcbi.1000609

Mortezapouraghdam, Z., Corona-Strauss, F. I., Takahashi, K., \& Strauss, D. J. (2018). Reducing the Effect of Spurious Phase Variations in Neural Oscillatory Signals. Frontiers in Computational Neuroscience, 12, 82. https://doi.org/10.3389/fncom.2018.00082

Muthukumaraswamy, S. D., \& Singh, K. D. (2011). A cautionary note on the interpretation of phase-locking estimates with concurrent changes in power. Clinical Neurophysiology, 122(11), 2324-2325. https://doi.org/10.1016/j.clinph.2011.04.003

Newson, J. J., \& Thiagarajan, T. C. (2019). EEG Frequency Bands in Psychiatric Disorders: A Review of Resting State Studies. Frontiers in Human Neuroscience, 12. https://doi.org/10.3389/fnhum.2018.00521

Neymotin, S. A., Barczak, A., O’Connell, M. N., McGinnis, T., Markowitz, N., Espinal, E., Griffith, E., Anwar, H., Dura-Bernal, S., Lytton, W. W., Jones, S. R., Bickel, S., \& Lakatos, P. (2020). Taxonomy of neural oscillation events in primate auditory cortex [Preprint]. bioRxiv. https://doi.org/10.1101/2020.04.16.045021

Neymotin, S. A., Daniels, D. S., Caldwell, B., McDougal, R. A., Carnevale, N. T., Jas, M., Moore, C. I., Hines, M. L., Hämäläinen, M., \& Jones, S. R. (2020). Human Neocortical Neurosolver (HNN), a new software tool for interpreting the cellular and network origin of human MEG/EEG data. ELife, 9, e51214. https://doi.org/10.7554/eLife.51214

Nikulin, V. V., Nolte, G., \& Curio, G. (2011). A novel method for reliable and fast extraction of neuronal EEG/MEG oscillations on the basis of spatio-spectral decomposition. Neurolmage, 55(4), 1528-1535. https://doi.org/10.1016/j.neuroimage.2011.01.057

Niso, G., Gorgolewski, K. J., Bock, E., Brooks, T. L., Flandin, G., Gramfort, A., Henson, R. N., Jas, M., Litvak, V., T. Moreau, J., Oostenveld, R., Schoffelen, J.-M., Tadel, F., Wexler, J., \& Baillet, S. (2018). MEG-BIDS, the brain imaging data structure extended to magnetoencephalography. Scientific Data, 5(1), 180110. https://doi.org/10.1038/sdata.2018.110

Nunez, P. L., \& Srinivasan, R. (2006). Electric fields of the brain: The neurophysics of EEG (2nd ed). Oxford University Press.

O’Neill, G. C., Tewarie, P., Vidaurre, D., Liuzzi, L., Woolrich, M. W., \& Brookes, M. J. (2018). Dynamics of large-scale electrophysiological networks: A technical review. Neurolmage, 180, 559-576. https://doi.org/10.1016/j.neuroimage.2017.10.003

Oostenveld, R., Fries, P., Maris, E., \& Schoffelen, J.-M. (2011). FieldTrip: Open Source Software for Advanced Analysis of MEG, EEG, and Invasive Electrophysiological Data. Computational Intelligence and Neuroscience, 2011, 1-9. 


\section{Oscillation Methods}

1344

1345

1346

1347

1348

1349

1350

1351

1352

1353

1354

1355

1356

1357

1358

1359

1360

1361

1362

1363

1364

1365

1366

1367

1368

1369

1370

1371

1372

1373

1374

1375

1376

1377

1378

1379

1380

1381

1382

1383

1384

1385

1386

1387

https://doi.org/10.1155/2011/156869

Palva, J. M., Wang, S. H., Palva, S., Zhigalov, A., Monto, S., Brookes, M. J., Schoffelen, J.-M., \& Jerbi, K. (2018). Ghost interactions in MEG/EEG source space: A note of caution on inter-areal coupling measures. Neurolmage, 173, 632-643.

https://doi.org/10.1016/j.neuroimage.2018.02.032

Palva, S., \& Palva, J. M. (2012). Discovering oscillatory interaction networks with M/EEG: Challenges and breakthroughs. Trends in Cognitive Sciences, 16(4), 219-230. https://doi.org/10.1016/j.tics.2012.02.004

Parra, L. C., Spence, C. D., Gerson, A. D., \& Sajda, P. (2005). Recipes for the linear analysis of EEG. Neurolmage, 28(2), 326-341. https://doi.org/10.1016/j.neuroimage.2005.05.032

Pascual-Marqui, R. D., Valdes-Sosa, P. A., \& Alvarez-Amador, A. (1988). A Parametric Model for Multichannel EEG Spectra. International Journal of Neuroscience, 40(1-2), 89-99. https://doi.org/10.3109/00207458808985730

Pernet, C. R., Appelhoff, S., Gorgolewski, K. J., Flandin, G., Phillips, C., Delorme, A., \& Oostenveld, R. (2019). EEG-BIDS, an extension to the brain imaging data structure for electroencephalography. Scientific Data, 6(1), 103. https://doi.org/10.1038/s41597-0190104-8

Pernet, C. R., Garrido, M. I., Gramfort, A., Maurits, N., Michel, C. M., Pang, E., Salmelin, R., Schoffelen, J. M., Valdes-Sosa, P. A., \& Puce, A. (2020). Issues and recommendations from the OHBM COBIDAS MEEG committee for reproducible EEG and MEG research. Nature Neuroscience, 23(12), 1473-1483. https://doi.org/10.1038/s41593-020-00709-0

Pesaran, B., Vinck, M., Einevoll, G. T., Sirota, A., Fries, P., Siegel, M., Truccolo, W., Schroeder, C. E., \& Srinivasan, R. (2018). Investigating large-scale brain dynamics using field potential recordings: Analysis and interpretation. Nature Neuroscience. https://doi.org/10.1038/s41593-018-0171-8

Pfurtscheller, G., \& Lopes da Silva, F. H. (1999). Event-related EEG/MEG synchronization and desynchronization: Basic principles. Clinical Neurophysiology, 110(11), 1842-1857. https://doi.org/10.1016/S1388-2457(99)00141-8

Piastra, M. C., Nüßing, A., Vorwerk, J., Clerc, M., Engwer, C., \& Wolters, C. H. (2020). A comprehensive study on electroencephalography and magnetoencephalography sensitivity to cortical and subcortical sources. Human Brain Mapping, hbm.25272. https://doi.org/10.1002/hbm.25272

Podvalny, E., Noy, N., Harel, M., Bickel, S., Chechik, G., Schroeder, C. E., Mehta, A. D., Tsodyks, M., \& Malach, R. (2015). A unifying principle underlying the extracellular field potential spectral responses in the human cortex. Journal of Neurophysiology, 114(1), 505-519. https://doi.org/10.1152/jn.00943.2014

Popov, T., Oostenveld, R., \& Schoffelen, J. M. (2018). FieldTrip Made Easy: An Analysis Protocol for Group Analysis of the Auditory Steady State Brain Response in Time, Frequency, and Space. Frontiers in Neuroscience, 12, 711. https://doi.org/10.3389/fnins.2018.00711

Quinn, A. J., van Ede, F., Brookes, M. J., Heideman, S. G., Nowak, M., Seedat, Z. A., Vidaurre, D., Zich, C., Nobre, A. C., \& Woolrich, M. W. (2019). Unpacking Transient Event Dynamics in Electrophysiological Power Spectra. Brain Topography, 32, 1020-1034. https://doi.org/10.1007/s10548-019-00745-5 


\section{Oscillation Methods}

1388

1389

1390

1391

1392

1393

1394

1395

1396

1397

1398

1399

1400

1401

1402

1403

1404

1405

1406

1407

1408

1409

1410

1411

1412

1413

1414

1415

1416

1417

1418

1419

1420

1421

1422

1423

1424

1425

1426

1427

1428

1429

1430

1431
Rey, H. G., Ahmadi, M., \& Quian Quiroga, R. (2015). Single trial analysis of field potentials in perception, learning and memory. Current Opinion in Neurobiology, 31, 148-155. https://doi.org/10.1016/j.conb.2014.10.009

Robertson, M. M., Furlong, S., Voytek, B., Donoghue, T., Boettiger, C. A., \& Sheridan, M. A. (2019). EEG Power Spectral Slope differs by ADHD status and stimulant medication exposure in early childhood. Journal of Neurophysiology, 122(6), 2427-2437. https://doi.org/10.1152/jn.00388.2019

Sameni, R., \& Seraj, E. (2017). A robust statistical framework for instantaneous electroencephalogram phase and frequency estimation and analysis. Physiological Measurement, 38(12), 2141-2163. https://doi.org/10.1088/1361-6579/aa93a1

Sanz Leon, P., Knock, S. A., Woodman, M. M., Domide, L., Mersmann, J., Mclntosh, A. R., \& Jirsa, V. (2013). The Virtual Brain: A simulator of primate brain network dynamics. Frontiers in Neuroinformatics, 7. https://doi.org/10.3389/fninf.2013.00010

Schaworonkow, N., Blythe, D. A. J., Kegeles, J., Curio, G., \& Nikulin, V. V. (2015). Power-law dynamics in neuronal and behavioral data introduce spurious correlations: Power-Law Dynamics in Neuronal and Behavioral Data. Human Brain Mapping, 36(8), 2901-2914. https://doi.org/10.1002/hbm.22816

Schaworonkow, N., \& Nikulin, V. V. (2019). Spatial neuronal synchronization and the waveform of oscillations: Implications for EEG and MEG. PLOS Computational Biology, 15(5), e1007055. https://doi.org/10.1371/journal.pcbi.1007055

Schoffelen, J.-M., \& Gross, J. (2009). Source connectivity analysis with MEG and EEG. Human Brain Mapping, 30(6), 1857-1865. https://doi.org/10.1002/hbm.20745

Sherman, M. A., Lee, S., Law, R., Haegens, S., Thorn, C. A., Hamalainen, M. S., Moore, C. I., \& Jones, S. R. (2016). Neural mechanisms of transient neocortical beta rhythms: Converging evidence from humans, computational modeling, monkeys, and mice. Proceedings of the National Academy of Sciences, 113(33), E4885-E4894. https://doi.org/10.1073/pnas.1604135113

Shin, H., Law, R., Tsutsui, S., Moore, C. I., \& Jones, S. R. (2017). The rate of transient beta frequency events predicts behavior across tasks and species. ELife, 6, e29086. https://doi.org/10.7554/eLife. 29086

Stokes, M., \& Spaak, E. (2016). The Importance of Single-Trial Analyses in Cognitive Neuroscience. Trends in Cognitive Sciences, 20(7), 483-486. https://doi.org/10.1016/j.tics.2016.05.008

Timmer, J., \& Konig, M. (1995). On Generating Power Law Noise. Astronomy and Astrophysics, $300,707-710$.

Urigüen, J. A., \& Garcia-Zapirain, B. (2015). EEG artifact removal-State-of-the-art and guidelines. Journal of Neural Engineering, 12(3), 031001. https://doi.org/10.1088/17412560/12/3/031001

van Diepen, R. M., \& Mazaheri, A. (2018). The Caveats of observing Inter-Trial PhaseCoherence in Cognitive Neuroscience. Scientific Reports, 8(1). https://doi.org/10.1038/s41598-018-20423-z

van Ede, F., Quinn, A. J., Woolrich, M. W., \& Nobre, A. C. (2018). Neural Oscillations: Sustained Rhythms or Transient Burst-Events? Trends in Neurosciences, 41(7), 415417. https://doi.org/10.1016/j.tins.2018.04.004 
1432

1433

1434

1435

1436

1437

1438

1439

1440

1441

1442

1443

1444

1445

1446

1447

1448

1449

1450

1451

1452

1453

1454

1455

1456

1457

1458

1459

1460

1461

1462

1463

1464

1465

1466

1467

1468

1469

1470

1471

1472

1473

1474

1475

van Vugt, M. K., Sederberg, P. B., \& Kahana, M. J. (2007). Comparison of spectral analysis methods for characterizing brain oscillations. Journal of Neuroscience Methods, 162(12), 49-63. https://doi.org/10.1016/j.jneumeth.2006.12.004

VanRullen, R. (2016). Perceptual Cycles. Trends in Cognitive Sciences, 20(10), 723-735. https://doi.org/10.1016/j.tics.2016.07.006

Varela, F. J., Lachaux, J.-P., Rodriguez, E., \& Martinerie, J. (2001). The brainweb: Phase synchronization and large-scale integration. Nature Reviews Neuroscience, 2(4), 229239. https://doi.org/10.1038/35067550

Vaz, A. P., Yaffe, R. B., Wittig, J. H., Inati, S. K., \& Zaghloul, K. A. (2017). Dual origins of measured phase-amplitude coupling reveal distinct neural mechanisms underlying episodic memory in the human cortex. Neurolmage, 148, 148-159. https://doi.org/10.1016/j.neuroimage.2017.01.001

Vidaurre, D., Quinn, A. J., Baker, A. P., Dupret, D., Tejero-Cantero, A., \& Woolrich, M. W. (2016). Spectrally resolved fast transient brain states in electrophysiological data. Neurolmage, 126, 81-95. https://doi.org/10.1016/j.neuroimage.2015.11.047

Voytek, B. (2016). The Virtuous Cycle of a Data Ecosystem. PLOS Computational Biology, 12(8), e1005037. https://doi.org/10.1371/journal.pcbi.1005037

Voytek, B., \& Knight, R. T. (2015). Dynamic Network Communication as a Unifying Neural Basis for Cognition, Development, Aging, and Disease. Biological Psychiatry, 77(12), 10891097. https://doi.org/10.1016/j.biopsych.2015.04.016

Voytek, B., Kramer, M. A., Case, J., Lepage, K. Q., Tempesta, Z. R., Knight, R. T., \& Gazzaley, A. (2015). Age-Related Changes in 1/f Neural Electrophysiological Noise. Journal of Neuroscience, 35(38), 13257-13265. https://doi.org/10.1523/JNEUROSCI.2332-14.2015

Wacker, M., \& Witte, H. (2013). Time-frequency Techniques in Biomedical Signal Analysis: A Tutorial Review of Similarities and Differences. Methods of Information in Medicine, 52(04), 279-296. https://doi.org/10.3414/ME12-01-0083

Wang, X.-J. (2010). Neurophysiological and Computational Principles of Cortical Rhythms in Cognition. Physiological Reviews, 90(3), 1195-1268. https://doi.org/10.1152/physrev.00035.2008

Waschke, L., Donoghue, T., Fiedler, L., Smith, S., Voytek, B., \& Obleser, J. (2021). Modalityspecific tracking of attention and sensory statistics in the human electrophysiological spectral exponent [Preprint]. bioRxiv. https://doi.org/10.1101/2021.01.13.426522

Watrous, A. J., \& Buchanan, R. J. (2020). The Oscillatory ReConstruction Algorithm adaptively identifies frequency bands to improve spectral decomposition in human and rodent neural recordings. Journal of Neurophysiology, 124(6), 1914-1922. https://doi.org/10.1152/jn.00292.2020

Watrous, A. J., Miller, J., Qasim, S. E., Fried, I., \& Jacobs, J. (2018). Phase-tuned neuronal firing encodes human contextual representations for navigational goals. ELife, 7 , e32554. https://doi.org/10.7554/eLife.32554

Wen, H., \& Liu, Z. (2016). Separating Fractal and Oscillatory Components in the Power Spectrum of Neurophysiological Signal. Brain Topography, 29(1), 13-26. https://doi.org/10.1007/s10548-015-0448-0

Wessel, J. R. (2020). $\beta$-Bursts Reveal the Trial-to-Trial Dynamics of Movement Initiation and Cancellation. The Journal of Neuroscience, 40(2), 411-423. 
1476

1477

1478

1479

1480

1481

1482

1483

1484

1485

1486

1487

1488

1489

1490

1491

1492

1493
https://doi.org/10.1523/JNEUROSCI.1887-19.2019

Whitten, T. A., Hughes, A. M., Dickson, C. T., \& Caplan, J. B. (2011). A better oscillation detection method robustly extracts EEG rhythms across brain state changes: The human alpha rhythm as a test case. Neurolmage, 54(2), 860-874.

https://doi.org/10.1016/j.neuroimage.2010.08.064

Widmann, A., Schröger, E., \& Maess, B. (2015). Digital filter design for electrophysiological data - a practical approach. Journal of Neuroscience Methods, 250, 34-46. https://doi.org/10.1016/j.jneumeth.2014.08.002

Wilson, G., Bryan, J., Cranston, K., Kitzes, J., Nederbragt, L., \& Teal, T. K. (2017). Good enough practices in scientific computing. PLOS Computational Biology, 13(6), e1005510. https://doi.org/10.1371/journal.pcbi.1005510

Wutz, A., Melcher, D., \& Samaha, J. (2018). Frequency modulation of neural oscillations according to visual task demands. Proceedings of the National Academy of Sciences, 115(6), 1346-1351. https://doi.org/10.1073/pnas.1713318115

Zich, C., Quinn, A. J., Mardell, L. C., Ward, N. S., \& Bestmann, S. (2020). Dissecting Transient Burst Events. Trends in Cognitive Sciences, 24(10), 784-788.

https://doi.org/10.1016/j.tics.2020.07.004 